\title{
ON THE SELMER GROUPS OF ABELIAN VARIETIES OVER FUNCTION FIELDS OF CHARACTERISTIC $p>0$
}

\author{
TADASHI OCHIAI AND FABIEN TRIHAN
}

\begin{abstract}
In this paper, we study a ( $p$-adic) geometric analogue for abelian varieties over a function field of characteristic $p$ of the cyclotomic Iwasawa theory and the noncommutative Iwasawa theory for abelian varieties over a number field initiated by Mazur and Coates respectively. We will prove some analogue of the principal results obtained in the case over a number field and we study new phenomena which did not happen in the case of number field case. We propose also a conjecture (Conjecture 1.6) which might be considered as a counterpart of the principal conjecture in the case over a number field.

This is a preprint which is distributed since 2005 which is still in the process of submision. Following a recent modification of some technical mistakes in the previous version of the paper as well as an amelioration of the presentation of the paper, we decide wider distribution via the archive.
\end{abstract}

\section{Contents}

1. Introduction

2. General abelian varieties over the base $F_{\infty}^{(p)}$

3. $\mu$-invariants of $X\left(A / F_{\infty}^{(p)}\right)$

4. A result over a $p$-adic Lie extension $L$

References

\section{INTRODUCTION}

Iwasawa theory was first initiated by Iwasawa who studied ideal class groups in the cyclotomic $\mathbb{Z}_{p}$-extension $K^{\text {cyc, }(p)}$ of a number field $K$ for a fixed prime $p$, which he regarded as an analogue of the extension of the coefficient field in the theory of algebraic curves over a finite field. He formulated the Iwasawa main conjecture, which predicts the equality between the algebraic $p$-adic $L$-function and the analytic $p$-adic $L$-function and was solved later by Mazur-Wiles [MW] and Wiles [W].

It was Mazur [Mz who first pointed out a plan of extending the philosophy of the Iwasawa theory to higher dimensional motives like elliptic curves over number fields in early 70's. Since then, the Iwasawa theory was generalized to ordinary motives and it seems to be extended to Selmer groups of Galois deformation spaces constructed by the theory of Mazur, Hida, Coleman, etc.

However, there is another direction of generalization called "non-commutative Iwasawa theory of elliptic curves" initiated by Coates.

Let us recall the classical (cyclotomic) Iwasawa theory for elliptic curves and its nonabelian generalization by Coates. Let $E$ be an elliptic curve over $K$. For any algebraic

The first author is supported by JSPS. The second author has been supported by the FNRS of Belgium. 
extension $L / K$, the Selmer group $\operatorname{Sel}(E / L)$ is defined to be

$$
\operatorname{Sel}(E / L)=\operatorname{Ker}\left[H_{\text {Gal }}^{1}\left(L, E_{p^{\infty}}\right) \rightarrow \prod_{v} H_{\text {Gal }}^{1}\left(L_{v}, E\left(\bar{L}_{v}\right)\right)\right],
$$

where $v$ runs over all primes of $L, E_{p}$ is the group of all $p$-power division points on $E$, and $H_{\text {Gal }}^{1}$ means the Galois cohomology. We denote by $X(E / L)$ the Pontrjagin dual $\operatorname{Hom}_{\text {cont }}\left(\operatorname{Sel}(E / L), \mathbb{Q}_{p} / \mathbb{Z}_{p}\right)$ of $\operatorname{Sel}(E / L)$. For the cyclotomic Iwasawa theory for elliptic curves, we consider $X\left(E / K^{\text {cyc, }(p)}\right)$ which is a compact $\mathbb{Z}_{p}\left[\left[\Gamma^{(p)}\right]\right]$-module where $\Gamma^{(p)}=$ $\operatorname{Gal}\left(K^{\text {cyc, }(p)} / K\right) \cong \mathbb{Z}_{p}$. It is not difficult to show that $X\left(E / K^{\text {cyc, }(p)}\right)$ is a finitely generated $\mathbb{Z}_{p}\left[\left[\Gamma^{(p)}\right]\right]$-module (cf. [Mz], $[\mathrm{Mn}]$ ).

Conjecture 1.1. Suppose that $E$ has good ordinary reduction at every primes of $K$ over p. Then $X\left(E / K^{\mathrm{cyc},(p)}\right)$ is a torsion $\mathbb{Z}_{p}\left[\left[\Gamma^{(p)}\right]\right]$-module.

Under this conjecture, the algebraic $p$-adic $L$-function $f_{E}^{\text {alg }} \in \mathbb{Z}_{p}\left[\left[\Gamma^{(p)}\right]\right]$ is defined to be the characteristic polynomial of $X\left(E / K^{\mathrm{cyc},(p)}\right)$ via the structure theorem of finitely generated $\mathbb{Z}_{p}\left[\left[\Gamma^{(p)}\right]\right]$-modules, which is roughly the product annihilators of the torsion $\mathbb{Z}_{p}\left[\left[\Gamma^{(p)}\right]\right]$ module $X\left(E / K^{\text {cyc, }(p)}\right)$ modulo its maximal pseudo-null $\mathbb{Z}_{p}\left[\left[\Gamma^{(p)}\right]\right]$-submodule. Here is the Iwasawa Main Conjecture in this case:

Conjecture 1.2 (Mazur). Suppose that E has good ordinary reduction at every primes of $K$ over $p$. Assuming the conjecture 1.1, The algebraic p-adic L-function $f_{E}^{\text {alg }}$ is equal to the analytic p-adic L-function $f_{E}^{\text {anal }} \in \mathbb{Z}_{p}\left[\left[\Gamma^{(p)}\right]\right]$ constructed by Mazur and Swinnerton-Dyer modulo multiplication by a unit of $\mathbb{Z}_{p}\left[\left[\Gamma^{(p)}\right]\right]$.

Remark 1.3. (1) When there is a prime lying over $p$ at which the elliptic curve $E$ has good supersingular reduction, it is known that $X\left(E / K^{\mathrm{cyc},(p)}\right)$ is not a torsion $\mathbb{Z}_{p}\left[\left[\Gamma^{(p)}\right]\right]$-module.

(2) By Euler system method Rubin [R] proves Conjecture 1.1 and Conjecture 1.2 for elliptic curves over $\mathbb{Q}$ with complex multiplication and Kato $[\mathrm{K}]$ proves Conjecture 1.1 and the divisibility $f_{E}^{\text {alg }} \mid f_{E}^{\text {anal }}$ of Conjecture 1.2 for large class of elliptic curves over $\mathbb{Q}$ without complex multiplication.

Recently, Skinner-Urban announces to have shown Conjecture 1.2 for large class of elliptic curves over $\mathbb{Q}$ without complex multiplication assuming the conjecture on the existence of Galois representations for the modular forms on $U(2,2)$.

From several reasons, Coates proposed to pursue the analogue of Conjecture 1.2 for non-commutative $p$-adic Lie extension $L$ of $K$. We recall two examples of such $p$-adic Lie extension $L$.

(1) Suppose that $K$ contains $p$-th root of unity $\zeta_{p}$. We take $L$ to be a Kummer extension of $K^{\mathrm{cyc},(p)}=\cup_{n \geq 1} K\left(\mu_{p^{n}}\right)$ defined by $L=\cup_{m \geq 1} K^{\mathrm{cyc},(p)}\left(\sqrt[p^{m}]{a}\right)$ for a fixed element $a \in K$. The Galois extension of $L / K$ has a non-split extension:

$$
0 \longrightarrow \mathbb{Z}_{p} \longrightarrow \operatorname{Gal}(L / K) \longrightarrow \mathbb{Z}_{p} \longrightarrow 0 \text {. }
$$

(2) Let $E$ be an elliptic curve over $K$ with no complex multiplication over a number field $\bar{K}$. Let $L:=K\left(E_{p^{\infty}}\right)$ be the field obtained by adjoining $E_{p \infty}$ to $K$. By Weil pairing, $L$ contains $K^{\text {cyc, }(p)}$. By a result of Serre, $\operatorname{Gal}(L / K)$ is identified with an open subgroup of $G L_{2}\left(\mathbb{Z}_{p}\right) \simeq \operatorname{Aut}\left(E_{p^{\infty}}\right)$. 
From now on through the paper, we will denote by $\Lambda(G)$ the algebra $\mathbb{Z}_{p}[[G]]$ for any profinite group $G$. For $G=\operatorname{Gal}(L / K), X(E / L)$ has a natural structure of a left $\Lambda(G)$ module. The algebra $\Lambda(G)$ is no more commutative, but have natural notion of "torsion $\Lambda(G)$-module". A non-commutative generalization of Conjecture 1.1 is known as follows:

Conjecture 1.4. Suppose that $E$ has good ordinary reduction at every primes of $K$ over p. Assume that $L$ contains $K^{\mathrm{cyc},(p)}$. Then $X(E / L)$ is a torsion $\Lambda(G)$-module.

To have an algebraic $p$-adic $L$-function is a necessary and important step for the study of Iwasawa theory. As in the classical cyclotomic case where $G \cong \mathbb{Z}_{p}$, we hope that the algebraic $p$-adic $L$-function is associated to $X(E / L)$ once Conjecture 1.4 is true. However, it is not impossible to associate a characteristic polynomial to a torsion $\Lambda(G)$ module when $G$ is non commutative. After rather negative results showing difficulties of non-commutative Iwasawa theory, CFKSV gave a convincing formalism of the Selmer group and the algebraic $p$-adic $L$-function inspired by the habilitation thesis of Venjakob at Heidelberg and based on calculation of K-groups of non-commutative Iwasawa algebras by Kato. Assuming that $G$ has a normal subgroup $H$ such that $G / H \cong \mathbb{Z}_{p}$, The important idea is that the $p$-adic $L$-function lives in a $K$-group $K_{1}\left(\Lambda(G)_{S^{*}}\right) / K_{1}(\Lambda(G))$, where $\Lambda(G)_{S^{*}}$ is the localization of $\Lambda(G)$ by a certain multiplicative set $S^{*}$. We refer the reader to [CFKSV] for how to choose $S^{*}$ and the basic results related to $S^{*}$. We remark that $S^{*}$ consists of every non-zero elements in $\Lambda(G)$ in the classical case $G=\mathbb{Z}_{p}$ and we have $K_{1}\left(\Lambda(G)_{S^{*}}\right) / K_{1}(\Lambda(G)) \cong \operatorname{Frac}(\Lambda(G))^{\times} / \Lambda(G)^{\times}$in this case. Since the characteristic power series is taken in a representative element of $\operatorname{Frac}(\Lambda(G))^{\times} / \Lambda(G)^{\times}$, it seems to be reasonable that the $p$-adic $L$-function should be constructed in $K_{1}\left(\Lambda(G)_{S^{*}}\right) / K_{1}(\Lambda(G)$ ). Under the existence of a closed subgroup $H$ with $G / H \cong \mathbb{Z}_{p}$, CFKSV gives an exact sequence:

$$
K_{1}(\Lambda(G)) \longrightarrow K_{1}\left(\Lambda(G)_{S^{*}}\right) \longrightarrow K_{0}\left(\mathfrak{M}_{H}\right)
$$

where $\mathfrak{M}_{H}$ is the category of finitely generated $\Lambda(G)$-modules $X$ such that $X / X(p)$ is finitely generated as $\Lambda(H)$-module, where $X(p)$ means the maximal $p$-power torsion submodule of $X$. According to [CFKSV], $\Lambda(G)$-modules which belong to $\mathfrak{M}_{H}$ satisfy all necessary properties which $p$-adic $L$-functions should have, related to the characteristic power series, the evaluation at characters of $G$ and the Euler-Poincaré characteristic. Hence, in order to have an algebraic $p$-adic $L$-function, we need the following conjecture stronger than Conjecture 1.4.

Conjecture 1.5. CFKSV] Assume that $E$ has ordinary reduction at every primes of $K$ over $p$. Then, $X(E / L) / X(E / L)(p)$ is a finitely generated $\Lambda(H)$-module.

In this paper, we intend to discuss an analogue of the commutative and non-commutative Iwasawa theory in the case of abelian varieties over a function field $F$ in one variable over a finite field $\mathbb{F}_{q}$. Let $A / F$ be an abelian variety. For any algebraic extension $K / F$, the Selmer group of $E / K$ in this context is by definition

$$
\operatorname{Sel}(A / K):=\operatorname{Ker}\left[H_{\mathrm{fl}}^{1}(K, A\{p\}) \longrightarrow \prod_{v} H_{\mathrm{fl}}^{1}\left(K_{v}, A\right)\right],
$$

where $A\{p\}$ denotes the $p$-divisible group associated to $A, H_{\mathrm{fl}}^{1}$ denotes the flat cohomology and $v$ runs all primes of $K$. 
(1) Let $F_{\infty}:=F \overline{\mathbb{F}}_{q}$, for an algebraic closure $\overline{\mathbb{F}}_{q}$ of $\mathbb{F}_{q}$. The Galois group $F_{\infty} / F$ is isomorphic to $\widehat{\mathbb{Z}}$. We will define $F_{\infty}^{(p)}$ to be $F_{\infty}^{(p)}=F \mathbb{F}_{q}^{(p)}$, where $\mathbb{F}_{q}^{(p)}$ is the unique subfield of $\overline{\mathbb{F}}_{q}$ such that $\operatorname{Gal}\left(\mathbb{F}_{q}^{(p)} / \mathbb{F}_{q}\right)$ is isomorphic to $\mathbb{Z}_{p}$.

(2) Let $F^{\text {sep }}$ be a fixed separable closure of $F$. It is known that the maximal pro- $p$ quotient of $\operatorname{Gal}\left(F^{\mathrm{sep}} / F\right)$ is a free pro- $p$ group of infinite rank. Hence any topologically finitely generated pro- $p$ group $G$ is realized as a Galois group $\operatorname{Gal}(L / F)$ of certain Galois extension.

Now we will state below our result in this paper. We denote by $X(A / L)$ the Pontrjagin dual of $\operatorname{Sel}(A / L)$, which has a natural structure of left $\Lambda(G)$-module. We define $Y(A / L)$ to be $X(A / L) / X(A / L)(p)$, which is again a left $\Lambda(G)$-module.

We propose the following conjecture:

Conjecture 1.6. Let $A$ be an abelian variety over $F$ with arbitrary reduction. Let $L$ be a Galois extension of $F$ containing $F_{\infty}^{(p)}$ whose Galois group $G=\operatorname{Gal}(L / F)$ is a finitely generated torsion-free pro-p group. We have the following conjecture.

(1) When $L$ is equal to $F_{\infty}^{(p)}, X(A / L)$ is a finitely generated torsion $\Lambda(G)$-module.

(2) When $L$ is an extension containing $F_{\infty}^{(p)}$ such that $\operatorname{Gal}(L / F)$ is a p-adic Lie extension, $Y(A / L)$ is a finitely generated $\Lambda(H)$-module where $H=\operatorname{Gal}\left(L / F_{\infty}^{(p)}\right)$.

Our main results over $F_{\infty}^{(p)}$ are as follows:

Theorem 1.7. Let $A$ be an abelian variety with arbitrary reduction. Then, $X\left(A / F_{\infty}^{(p)}\right)$ is a finitely generated torsion $\Lambda(G)$-module for $G=\operatorname{Gal}\left(F_{\infty}^{(p)} / F\right)$.

Theorem 1.7 will be proved in $\S 3$. We insist that the independence of the reduction of $A$ for $X\left(A / F_{\infty}^{(p)}\right)$ to be torsion over $\Lambda(G)$ is a different phenomenon when we compare our situation to that of the case of abelian varieties over the cyclotomic tower $K^{\text {cyc, }(p)}$ of a number field $K$ (cf. Conjecture 1.1).

For a torsion $\Lambda(G)$-module $M$ with $G \cong \mathbb{Z}_{p}$, we denote by $\mu(M)$ the length of $M_{(p)}$ over $\Lambda(G)_{(p)}$, where ()$_{(p)}$ means the localization at the height-one prime ideal $(p)$. The invariant $\mu(M)$ is called the $\mu$-invariant of $M$ and $\mu(M)$ is an important numerical invariant of $M$. It is known that $\mu\left(X\left(A / K^{\mathrm{cyc},(p)}\right)\right)$ is strongly dependent of the mod- $p$ representation of $A$. As the following result shows, the the $\mu$-invariant $\mu\left(X\left(A / F_{\infty}^{(p)}\right)\right)$ for an abelian variety $A$ over a function field $F$ seems to be strongly influenced by the reduction type of A.

Theorem 1.8. (1) Assume that there exists a finite separable extension $F^{\prime}$ of $F$ which satisfies one of the following conditions:

(OF) $A \times_{F} F^{\prime}$ is isomorphic to an ordinary abelian variety defined over a finite field.

(SF) $A \times_{F} F^{\prime}$ is isomorphic to a supersingular abelian variety defined over a finite field and the proper smooth curve $C_{F^{\prime}}$ which is the model of $F^{\prime}$ has invertible Hasse-Witt matrix.

Then, we have $\mu\left(X\left(A / F_{\infty}^{(p)}\right)\right)=0$.

(2) Let $A$ be an isotrivial abelian variety having everywhere supersingular reduction. We have $\mu\left(X\left(A / F_{\infty}^{(p)}\right)\right)=0$ if and only if there exists a finite extension $F^{\prime} / F$ such 
that the Hasse-Witt matrix for $F^{\prime}$ is invertible and that $A \times_{F} F^{\prime}$ is isomorphic to an abelian variety defined over a finite field.

Theorem 1.8 will be proved in $§ 3$. The following results give an evidence for Conjecture 1.6 .

Theorem 1.9. Let $L$ be a Galois extension of $F$ containing $F_{\infty}^{(p)}$ whose Galois group $G=\operatorname{Gal}(L / F)$ is a p-adic Lie group with no non-trivial torsion elements. Let $A$ be an abelian variety over $F$. We denote by $H$ the Galois group $\operatorname{Gal}\left(L / F_{\infty}^{(p)}\right)$. Let us assume the following conditions:

(1) $L / F_{\infty}^{(p)}$ is ramified at only finitely many primes of $F_{\infty}^{(p)}$, say $S$.

(2) A has good reduction outside $S$. For each prime $v \in S$, A has ordinary reduction (not necessarily good ordinary reduction).

(3) We have $\mu\left(X\left(A / F_{\infty}^{(p)}\right)\right)=0$.

Then, $X(A / L)$ is a finitely generated $\Lambda(H)$-module.

Theorem 1.9 will be proved in $\S 4$.

Corollary 1.10. Assume that there exists a finite separable extension $F^{\prime}$ satisfying the condition ( $\mathrm{OF})$. Then, $X(A / L)$ is a finitely generated $\Lambda(H)$-module.

In this paper, we decided not to prove our result in full generality. However, in proving our results, the necessary hypothesis on the reduction of the abelian variety was rather general as compared to the cases of abelian varieties over number fields. Thus, Main Conjecture (Conjecture 1.6) was stated under more general condition than the one we imagined at the beginning of our work. In subsequent papers, we will generalize our results by removing some of hypothesis assumed in our theorems and we would like to make further research which is expected from the philosophy of the Iwasawa theory.

Acknowledgements. The first author would like to thank Yoshitaka Hachimori for useful discussion on non-commutative generalization of Iwasawa theory. The second author would like to thank Kazuya Kato for introducing him to the theory of Iwasawa and Takeshi Saito and the University of Tokyo for their hospitality. The authors are grateful to Kato for pointing out technical mistakes in the first draft of the paper. They are also grateful to anonymous refrees for pointing out historical mistakes and technical mistakes in 82 .

\section{General abelian varieties over the Base $F_{\infty}^{(p)}$}

In this section, we will prove Theorem 1.7. First, we will need the following lemma:

Lemma 2.1. Let $F^{\prime} / F$ be a finite Galois extension. We denote by $A^{\prime}$ the extension $A^{\prime}=A \times_{F} F^{\prime}$. If $X\left(A^{\prime} / F_{\infty}^{\prime(p)}\right)$ is a finitely generated torsion $\Lambda\left(G^{\prime}\right)$-module for $G^{\prime}=$ $\operatorname{Gal}\left(F_{\infty}^{\prime,(p)} / F^{\prime}\right), X\left(A / F_{\infty}^{(p)}\right)$ is a finitely generated torsion $\Lambda(G)$-module for $G=\operatorname{Gal}\left(F_{\infty}^{(p)} / F\right)$. 
Proof. Suppose that $X\left(A^{\prime} / F_{\infty}^{\prime}(p)\right)$ is a finitely generated torsion $\Lambda\left(G^{\prime}\right)$-module. Then we have $J=\operatorname{Gal}\left(F^{\prime} / F_{\infty}^{(p)} \cap F^{\prime}\right) \cong \operatorname{Gal}\left(F_{\infty}^{\prime,(p)} / F_{\infty}^{(p)}\right)$.

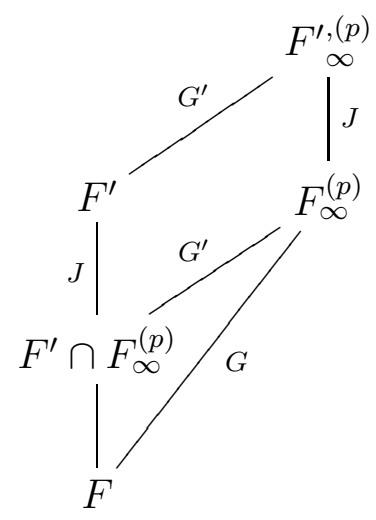

Recall that $X\left(A / F_{\infty}^{(p)}\right)$ and $X\left(A^{\prime} / F_{\infty}^{\prime,(p)}\right)$ are quotients of the modules $H_{\mathrm{f}}^{1}\left(F_{\infty}^{(p)}, A\{p\}\right)^{\vee}$ and $H_{\mathrm{fl}}^{1}\left(F_{\infty}^{\prime}(p), A\{p\}\right)^{\vee}$ respectively by the definition given in (1). Hence the cokernel of the natural $\Lambda\left(G^{\prime}\right)$-linear map $X\left(A^{\prime} / F_{\infty}^{\prime(p)}\right)_{J} \longrightarrow X\left(A / F_{\infty}^{(p)}\right)$ is a quotient of the Pontrjagin dual of a finite group $H^{1}\left(J, A\{p\}\left(F_{\infty}^{\prime,(p)}\right)\right) \cong \operatorname{Ker}\left[H_{\mathrm{fl}}^{1}\left(F_{\infty}^{(p)}, A\{p\}\right) \longrightarrow H_{\mathrm{fl}}^{1}\left(F_{\infty}^{\prime,(p)}, A\{p\}\right)^{J}\right]$. Hence the $\Lambda(G)$-module $X\left(A / F_{\infty}^{(p)}\right)$ is a torsion $\Lambda\left(G^{\prime}\right)$-module via natural identification of $G^{\prime}$ as an open subgroup of $G$. Hence it is also torsion over $\Lambda(G)$.

By the semi-stable reduction theorem for abelian varieties, an abelian variety $A$ over $F$ has everywhere semi-stable reduction after the base field extension $A \times_{F} F^{\prime}$ by a certain finite Galois extension $F^{\prime} / F$. Hence, by Lemma 2.1, we may (and we will) assume the following condition (SS) from now on through this section for the proof of Theorem 1.7;

(SS) $A / F$ has everywhere semi-stable reduction.

Let $C_{F_{\infty}}$ be a proper smooth geometrically connected curve over $\overline{\mathbb{F}}_{q}$ which is the model of the function field $F_{\infty}=F \overline{\mathbb{F}}_{q}$. We denote by $U$ the dense open subset of $C_{F_{\infty}}$ such that $A \times{ }_{F} F_{\infty}$ has good reduction on $U$ and we denote by $Z$ the complement $C_{F_{\infty}} \backslash U$. Let $\mathcal{A}$ be the Néron model of $A \times{ }_{F} F_{\infty}$ over $C_{F_{\infty}}$. We associate the Lie algebra $\operatorname{Lie}(\mathcal{A})$ to $\mathcal{A}$, which is a sheaf of algebras on $C_{F_{\infty}}$.

In [K-T], syntomic cohomology for abelian varieties has been studied for application to the Birch and Swinnerton-Dyer conjecture of abelian varieties over function fields. In this section, we will reduce our main result to Theorem 2.6 for general Dieudonné crystals using tools studied in [K-T] and give a proof of Theorem 2.6.

We shall summarize necessary results and definition for later use in this section. From now on, we denote the Witt algebra $W\left(\overline{\mathbb{F}}_{q}\right)$ by $W$ if there is no confusion. Let us denote by $C_{F_{\infty}}^{\sharp}$ the $\log$ scheme associated to a divisor $Z$ on $C_{F_{\infty}}$. We refer the reader to [K-T] for detailed explanation. Let $D$ be a Dieudonné crystal on $C_{F_{\infty}}^{\sharp} / W$. If $Z$ is empty, then $D$ is a classical Dieudonné crystal corresponding to a $p$-divisible group $G / C_{F_{\infty}}$. Recall the following theorem from $[\mathrm{K}-\mathrm{T}]$ :

Theorem 2.2. Let $D$ be a Dieudonné crystal on $C_{F_{\infty}}^{\sharp}$. Let $i$ be the canonical morphism of topos of [BBM] from the topos of sheaves on $\left(C_{F_{\infty}}\right)_{\text {ét }}$ to the crystalline topos $\left(C_{F_{\infty}}^{\sharp} / W\right)_{\text {crys }}$. There exists an $\mathcal{O}_{C_{F_{\infty}}}$-module $\operatorname{Lie}(D)$ locally free of finite rank and a surjective map of sheaves $D \rightarrow i_{*}(\operatorname{Lie}(D))$ in $\left(C_{F_{\infty}}^{\sharp} / W\right)_{\text {crys }}$. 
Proof. The proof of [K-T, §5.3], remains still valid if we replace a base scheme over $\mathbb{F}_{q}$ by a scheme over $\overline{\mathbb{F}}_{q}$.

Let us summarize necessary results and definitions related to the above theorem.

(1) We denote by $D^{0}$ the kernel of $D \longrightarrow i_{*}(\operatorname{Lie}(D))$ in $\left(C_{F_{\infty}}^{\sharp} / W\right)_{\text {crys }}$ and we denote the canonical injection by $1: D^{0} \longrightarrow D$. By applying the canonical projection $u_{*}$ from the crystalline topos $\left(C_{F_{\infty}}^{\sharp} / W\right)_{\text {crys }}$ to the topos of sheaves on $\left(C_{F_{\infty}}\right)_{\text {ét }}$, we get a distinguished triangle:

$$
R u_{*} D^{0} \stackrel{1}{\longrightarrow} R u_{*} D \longrightarrow \operatorname{Lie}(D) .
$$

We can twist this triangle by the divisor $Z$ to get a triangle:

$$
R u_{*} D^{0}(-Z) \stackrel{1}{\longrightarrow} R u_{*} D(-Z) \longrightarrow \operatorname{Lie}(D)(-Z) .
$$

where $D(-Z)$ is the twist of the Dieudonné crystal $D$ defined in $[\mathrm{K}-\mathrm{T}, \S 5.11]$.

(2) We denote the $i$-th cohomology associated to $R u_{*} D^{0}$ (resp. $R u_{*} D$ ) by the symbol $H_{\text {crys }}^{i}\left(C_{F_{\infty}}^{\sharp} / W, D^{0}(-Z)\right)\left(\right.$ resp. $\left.H_{\text {crys }}^{i}\left(C_{F_{\infty}}^{\sharp} / W, D(-Z)\right)\right)$ and by $H_{\text {crys }}^{i}\left(C_{F_{\infty}}^{\sharp} / W, D^{0}(-Z) \otimes\right.$ $\left.\mathbb{Q}_{p} / \mathbb{Z}_{p}\right)$ (resp. $\left.H_{\text {crys }}^{i}\left(C_{F_{\infty}}^{\sharp} / W, D(-Z) \otimes \mathbb{Q}_{p} / \mathbb{Z}_{p}\right)\right)$ the $i$-th cohomology associated to $R u_{*} D^{0} \otimes^{\mathbb{L}} \mathbb{Q}_{p} / \mathbb{Z}_{p}$ (resp. $R u_{*} D \otimes^{\mathbb{L}} \mathbb{Q}_{p} / \mathbb{Z}_{p}$ ) by abuse of notation. We have the following lemma:

\section{Lemma 2.3.}

(a) The canonical map $H_{\text {crys }}^{i}\left(C_{F_{\infty}}^{\sharp} / W, D^{0}(-Z)\right) \stackrel{1}{\longrightarrow} H_{\text {crys }}^{i}\left(C_{F_{\infty}}^{\sharp} / W, D(-Z)\right)$ has a kernel and a cokernel killed by $p$.

(b) $H_{\text {crys }}^{i}\left(C_{F_{\infty}}^{\sharp} / W, D^{0}(-Z)\right)$ and $H_{\text {crys }}^{i}\left(C_{F_{\infty}}^{\sharp} / W, D(-Z)\right)$ are finitely generated $W$ modules with the same rank.

Proof. By taking the cohomology of the triangle (3), we have the exact sequence:

$$
\begin{aligned}
H_{\mathrm{fl}}^{i-1}\left(C_{F_{\infty}}, \operatorname{Lie}(D)(-Z)\right) \longrightarrow & H_{\mathrm{crys}}^{i}\left(C_{F_{\infty}}^{\sharp} / W, D^{0}(-Z)\right) \\
& \stackrel{\mathbf{1}}{\longrightarrow} H_{\mathrm{crys}}^{i}\left(C_{F_{\infty}}^{\sharp} / W, D(-Z)\right) \longrightarrow H_{\mathrm{fl}}^{i}\left(C_{F_{\infty}}, \operatorname{Lie}(D)(-Z)\right) .
\end{aligned}
$$

Since $H_{\mathrm{fl}}^{j}\left(C_{F_{\infty}}, \operatorname{Lie}(D)(-Z)\right)$ is a finite dimensional vector space over $\overline{\mathbb{F}}_{q}$ for every $j$, this completes the proof of the first assertion. The second assertion follows from the first one and the fact that crystalline cohomologies over a proper log smooth base scheme with finite locally free coefficients are of finite dimension by Tsuji [T].

(3) As in [K-T, §5.8], we can construct a Frobenius operator

$$
\varphi: R u_{*} D^{0}(-Z) \longrightarrow R u_{*} D(-Z),
$$

which induces a $\sigma$-linear homomorphism

$$
\varphi_{i}: H_{\text {crys }}^{i}\left(C_{F_{\infty}}^{\sharp} / W, D^{0}(-Z)\right) \longrightarrow H_{\text {crys }}^{i}\left(C_{F_{\infty}}^{\sharp} / W, D(-Z)\right)
$$

for each $i$ by $\sigma$-linearity of the composed map $F \circ \iota: D \rightarrow D$, where $\iota: D \rightarrow \sigma^{*} D$ is the map sending $x \rightarrow 1 \otimes x$. 
(4) We denote $\mathcal{S}_{D}$ the mapping fiber of the map

$$
\mathbf{1}-\varphi: R u_{*} D^{0}(-Z) \longrightarrow R u_{*} D(-Z) .
$$

This complex is an object in the derived category of complexes of sheaves over $\left(C_{F_{\infty}}\right)$ ét and we have a distinguished triangle:

$$
\mathcal{S}_{D} \longrightarrow R u_{*} D^{0}(-Z) \stackrel{1-\varphi}{\longrightarrow} R u_{*} D(-Z) .
$$

We denote by $H_{\text {syn }}^{i}\left(C_{F_{\infty}}, \mathcal{S}_{D}\right)$ the i-th cohomology group of $R \Gamma\left(C_{F_{\infty}}, \mathcal{S}_{D}\right)$ and by $H_{\text {syn }}^{i}\left(C_{F_{\infty}}, \mathcal{S}_{D} \otimes \mathbb{Q}_{p} / \mathbb{Z}_{p}\right)$ the i-th cohomology group of $R \Gamma\left(C_{F_{\infty}}, \mathcal{S}_{D}\right) \otimes_{\mathbb{Z}_{p}}^{\mathbb{Q}} \mathbb{Q}_{p} / \mathbb{Z}_{p}$ by abuse of notation.

(5) Finally, let $X\left(D / F_{\infty}\right)$ denote the Pontrjagin dual of $H_{\mathrm{syn}}^{i}\left(C_{F_{\infty}}, \mathcal{S}_{D} \otimes \mathbb{Q}_{p} / \mathbb{Z}_{p}\right)$ and let $Y\left(D / F_{\infty}\right)=X\left(D / F_{\infty}\right) / X\left(D / F_{\infty}\right)(p)$.

In this paper, we are concerned with the two following crucial examples of Dieudonné crystals:

Example 2.4. (1) Let $A / F$ be an abelian variety satisfying the condition (SS). We denote $U$ the dense open subset of $C_{F_{\infty}}$ such that $A / F$ has good reduction on $U$ and $Z:=C_{F_{\infty}} \backslash U$. Let $\mathcal{A}$ be the Néron model of $A \times_{F} F_{\infty}$ over $C_{F_{\infty}}$. It has been shown in [K-T, §4.9] that the classical Dieudonné crystal $D(\mathcal{A} / U)$ extends to a Dieudonné crystal $D(\mathcal{A})$ over $C_{F_{\infty}}^{\sharp} / W$.

(2) For any p-divisible group $\mathcal{G}$ over $C_{F_{\infty}}$, we can associate the classical Dieudonné crystal $D(\mathcal{G})$ which is a crystal over $C_{F_{\infty}} / W$.

In the rest of this section, we are going to deduce "finiteness theorems" over $F_{\infty}^{(p)}$ from "finiteness theorems" over $F_{\infty}$ introduced above. We denote by $\Gamma\left(\operatorname{resp} . \Gamma^{(p)}, \Gamma^{\left(p^{\prime}\right)}\right)$ the Galois group $\operatorname{Gal}\left(F_{\infty} / F\right)$ (resp. $\operatorname{Gal}\left(F_{\infty}^{(p)} / F\right), \operatorname{Gal}\left(F_{\infty} / F_{\infty}^{(p)}\right)$ ). Note that the group $\Gamma$ (resp. $\left.\Gamma^{(p)}, \Gamma^{\left(p^{\prime}\right)}\right)$ is isomorphic to $\widehat{\mathbb{Z}}$ (resp. $\left.\mathbb{Z}_{p}, \prod_{l \neq p} \mathbb{Z}_{l}\right)$. Note that $W=W\left(\overline{\mathbb{F}}_{q}\right)$ is naturally endowed with a structure of $\Lambda(\Gamma)=\mathbb{Z}_{p}[[\Gamma]]$-module. In fact $W\left(\mathbb{F}_{q^{n}}\right)$ is naturally a $\Lambda(\Gamma)$ module through the action via a unique quotient $\Gamma_{n}$ of $\Gamma$ isomorphic to $\mathbb{Z} /(n)$. Hence $\lim _{\rightarrow n} W\left(\mathbb{F}_{q^{n}}\right)$ is also a $\Lambda(\Gamma)$-module. Since $W$ is the $p$-adic completion of $\lim _{\rightarrow n} W\left(\mathbb{F}_{q^{n}}\right)$, we see that $W$ is a $\Lambda(\Gamma)$-module. By crystalline base change theorem, We have:

$$
H_{\text {crys }}^{i}\left(C_{F_{\infty}}^{\sharp} / W, E\right) \cong H_{\text {crys }}^{i}\left(C_{F}^{\sharp} / W\left(\mathbb{F}_{q}\right), E\right) \otimes_{W\left(\mathbb{F}_{q}\right)} W
$$

for $E=D^{0}(-Z)$ or $E=D(-Z)$. Hence $H_{\text {crys }}^{i}\left(C_{F_{\infty}}^{\sharp} / W, E\right)$ is naturally endowed with a structure of $\Lambda(\Gamma)$-module. Since the triangle (4) is also naturally obtained as the $p$-adic completion of the inductive limit of the similar triangles over $W\left(\mathbb{F}_{q^{n}}\right)$, we also show that the syntomic cohomology is endowed with a structure of $\Lambda(\Gamma)$-module and the long exact sequence induced from the triangle (41) is $\Lambda(\Gamma)$-linear.

Though the Iwasawa algebra $\Lambda(\Gamma)$ is neither integral nor Noetherian, the algebra $\Lambda\left(\Gamma^{(p)}\right)$ is an integral Noetherian domain. Thus, we recall the following fundamental facts without proof:

Lemma 2.5. Let $M^{\prime} \longrightarrow M \longrightarrow M^{\prime \prime}$ be an exact sequence of $\Lambda\left(\Gamma^{(p)}\right)$-modules. Then, if two of these three modules are torsion of finite type over $\Lambda\left(\Gamma^{(p)}\right)$, the other one is also torsion of finite type over $\Lambda\left(\Gamma^{(p)}\right)$. In other words, the category of finite type torsion $\Lambda\left(\Gamma^{(p)}\right)$-modules are stable under taking a submodule, a quotient and an extension in the category of $\Lambda\left(\Gamma^{(p)}\right)$-modules. 
We use the following proposition for the proof of Theorem 1.7 at the end of this section:

Proposition 2.6. Let $D$ be a Dieudonné crystal over $C_{F_{\infty}}^{\sharp} / W$. Then $X\left(D / F_{\infty}\right)_{\Gamma\left(p^{\prime}\right)}$ is a finitely generated torsion $\Lambda\left(\Gamma^{(p)}\right)$-module.

Recall that the following exact sequence:

$$
\begin{aligned}
\cdots \longrightarrow H_{\mathrm{syn}}^{1}\left(C_{F_{\infty}}, \mathcal{S}_{D}\right) \otimes \mathbb{Q}_{p} \stackrel{\alpha}{\longrightarrow} & H_{\mathrm{syn}}^{1}\left(C_{F_{\infty}}, \mathcal{S}_{D} \otimes \mathbb{Q}_{p} / \mathbb{Z}_{p}\right) \\
& \stackrel{\beta}{\longrightarrow} H_{\mathrm{syn}}^{2}\left(C_{F_{\infty}}, \mathcal{S}_{D}\right) \stackrel{\gamma}{\longrightarrow} H_{\mathrm{syn}}^{2}\left(C_{F_{\infty}}, \mathcal{S}_{D}\right) \otimes \mathbb{Q}_{p} \longrightarrow \cdots
\end{aligned}
$$

induces a short exact sequence

$$
0 \longrightarrow \operatorname{Im}(\alpha) \longrightarrow H_{\text {syn }}^{1}\left(C_{F_{\infty}}, \mathcal{S}_{D} \otimes \mathbb{Q}_{p} / \mathbb{Z}_{p}\right) \longrightarrow \operatorname{Im}(\beta) \longrightarrow 0 .
$$

By taking the Pontrjagin dual of (15), we have

$$
0 \longrightarrow \operatorname{Im}(\beta)^{\vee} \longrightarrow X\left(D / F_{\infty}\right) \longrightarrow \operatorname{Im}(\alpha)^{\vee} \longrightarrow 0
$$

Now, note that taking the $\Gamma^{\left(p^{\prime}\right)}$-coinvariant of a module over $\Lambda(\Gamma) \cong \Lambda\left(\Gamma^{(p)}\right) \widehat{\otimes}_{\mathbb{Z}_{p}} \Lambda\left(\Gamma^{\left(p^{\prime}\right)}\right) \cong$ $\Lambda\left(\Gamma^{(p)}\right)\left[\left[\Gamma^{\left(p^{\prime}\right)}\right]\right]$ is equal to taking the base extension of the module by $\otimes_{\Lambda(\Gamma)} \Lambda\left(\Gamma^{(p)}\right)$. Since taking the $\Gamma^{\left(p^{\prime}\right)}$-coinvariant is a left exact functor, the sequence (5) gives us the following:

$$
\left(\operatorname{Im}(\beta)^{\vee}\right)_{\Gamma^{\left(p^{\prime}\right)}} \longrightarrow X\left(D / F_{\infty}\right)_{\Gamma^{\left(p^{\prime}\right)}} \longrightarrow\left(\operatorname{Im}(\alpha)^{\vee}\right)_{\Gamma^{\left(p^{\prime}\right)}} \longrightarrow 0,
$$

where the modules and the morphisms are naturally defined over $\Lambda\left(\Gamma^{(p)}\right)$. Thanks to Lemma 2.5 and the short exact sequence (7), it is enough to prove that $\left(\operatorname{Im}(\alpha)^{\vee}\right)_{\Gamma\left(p^{\prime}\right)}$ and $\left(\operatorname{Im}(\beta)^{\vee}\right)_{\Gamma^{\left(p^{\prime}\right)}}$ are torsion $\Lambda\left(\Gamma^{(p)}\right)$-modules of finite type in order to prove Proposition 2.6. Thus, Proposition 2.6 follows from Corollary 2.9 and Lemma 2.10 which will be shown below.

Lemma 2.7. $H_{\mathrm{syn}}^{1}\left(C_{F_{\infty}}, \mathcal{S}_{D}\right) \otimes \mathbb{Q}_{p}$ is a finite dimensional $\mathbb{Q}_{p}$-vector space.

Proof. The long exact sequence

$$
\begin{aligned}
\left.\cdots \longrightarrow H_{\mathrm{syn}}^{i}\left(C_{F_{\infty}}, \mathcal{S}_{D}\right) \otimes \mathbb{Q}_{p} \longrightarrow H_{\mathrm{crys}}^{i}\left(C_{F_{\infty}}^{\sharp} / W, D^{0}(-Z)\right)\right) \otimes \mathbb{Q}_{p} & \stackrel{1-\varphi_{i}}{\longrightarrow} H_{\mathrm{crys}}^{i}\left(C_{F_{\infty}}^{\sharp} / W, D(-Z)\right) \otimes \mathbb{Q}_{p} \longrightarrow \cdots
\end{aligned}
$$

can be rewritten

$$
\begin{aligned}
\cdots \longrightarrow H_{\mathrm{syn}}^{i}\left(C_{F_{\infty}}, \mathcal{S}_{D}\right) \otimes \mathbb{Q}_{p} & \longrightarrow H_{\mathrm{crys}}^{i}\left(C_{F_{\infty}}^{\sharp} / W, D(-Z)\right) \otimes \mathbb{Q}_{p} \\
& \stackrel{1-\varphi_{i}}{\longrightarrow} H_{\mathrm{crys}}^{i}\left(C_{F_{\infty}}^{\sharp} / W, D(-Z)\right) \otimes \mathbb{Q}_{p} \longrightarrow \cdots
\end{aligned}
$$

thanks to Lemma 2.3.

We deduce from this long exact sequence the following short exact sequence:

$$
0 \longrightarrow \operatorname{Coker}\left(1-\varphi_{0}\right) \rightarrow H_{\text {syn }}^{1}\left(C_{F_{\infty}}, \mathcal{S}_{D}\right) \otimes \mathbb{Q}_{p} \longrightarrow \operatorname{Ker}\left(1-\varphi_{1}\right) \longrightarrow 0
$$

Since $H_{\text {crys }}^{i}\left(C_{F_{\infty}}^{\sharp} / W, D(-Z)\right) \otimes \mathbb{Q}_{p}$ is a finite dimensional $P_{0^{-}}$-vector space, with $P_{0}=$ $\operatorname{Frac}(W)$, the assertion results from the following classical result of which we omit the proof:

Lemma 2.8. Let $V$ be a finite dimensional $P_{0}$-vector space endowed with a $\sigma$-linear operator $\varphi: V \longrightarrow V$. Then $1-\varphi$ is a surjective map whose kernel is a finite dimensional $\mathbb{Q}_{p}$-vector space. 
Corollary 2.9. The Pontrjagin dual $\operatorname{Im}(\alpha)^{\vee}$ of $\operatorname{Im}(\alpha)$ is a free $\mathbb{Z}_{p}$-module whose rank is equal to $\operatorname{dim}_{\mathbb{Q}_{p}}\left(H_{\text {syn }}^{1}\left(C_{F_{\infty}}, \mathcal{S}_{D}\right) \otimes \mathbb{Q}_{p}\right)$. In particular, $\left(\operatorname{Im}(\alpha)^{\vee}\right)_{\Gamma^{\left(p^{\prime}\right)}}$ is a finitely generated torsion $\Lambda\left(\Gamma^{(p)}\right)$-module.

Proof of Corollary 2.9. By definition of the syntomic complex we have a long exact sequence

$$
\begin{aligned}
\cdots \longrightarrow H_{\mathrm{syn}}^{i}\left(C_{F_{\infty}}, \mathcal{S}_{D} \otimes \mathbb{Q}_{p} / \mathbb{Z}_{p}\right) \longrightarrow H_{\mathrm{crys}}^{i}\left(C_{F_{\infty}}^{\sharp} / W, D^{0}(-Z) \otimes \mathbb{Q}_{p} / \mathbb{Z}_{p}\right) & \\
\qquad & \stackrel{1-\varphi_{i}}{\longrightarrow} H_{\mathrm{crys}}^{i}\left(C_{F_{\infty}}^{\sharp} / W, D(-Z) \otimes \mathbb{Q}_{p} / \mathbb{Z}_{p}\right) \longrightarrow \cdots
\end{aligned}
$$

where the middle and right handside modules are torsion. In particular, $H_{\mathrm{syn}}^{1}\left(C_{F_{\infty}}, \mathcal{S}_{D} \otimes\right.$ $\left.\mathbb{Q}_{p} / \mathbb{Z}_{p}\right)$ is a torsion $\mathbb{Z}_{p}$-module. Thus, $\operatorname{Im}(\alpha)$ is a torsion $\mathbb{Z}_{p}$-module which is a quotient of $H_{\text {syn }}^{1}\left(C_{F_{\infty}}, \mathcal{S}_{D}\right) \otimes \mathbb{Q}_{p}$. We deduce from 2.7, that $\operatorname{Im}(\alpha)$ is cofree of finite rank $n \leq \operatorname{dim}_{\mathbb{Q}_{p}}\left(H_{\text {syn }}^{1}\left(C_{F_{\infty}}, \mathcal{S}_{D}\right) \otimes \mathbb{Q}_{p}\right)$. But since $H_{\text {syn }}^{1}\left(C_{F_{\infty}}, \mathcal{S}_{D}\right)$ is an extension of a submodule of $H_{\text {crys }}^{1}\left(C_{F_{\infty}}^{\sharp} / W, D^{0}(-Z)\right)$ by a quotient of $H_{\text {crys }}^{0}\left(C_{F_{\infty}}^{\sharp} / W, D(-Z)\right)$, $\operatorname{Ker}(\alpha)$ contains no non-zero $p$-divisible elements. This proves that the corank of $\operatorname{Im}(\alpha)$ is equal to $\operatorname{dim}_{\mathbb{Q}_{p}}\left(H_{\text {syn }}^{1}\left(C_{F_{\infty}}, \mathcal{S}_{D}\right) \otimes \mathbb{Q}_{p}\right)$.

We now study the term $\operatorname{Im}(\beta)$ :

Lemma 2.10. $\left(\operatorname{Im}(\beta)^{\vee}\right)_{\Gamma^{\left(p^{\prime}\right)}}$ is a finitely generated torsion $\Lambda\left(\Gamma^{(p)}\right)$-module.

Proof of Lemma 2.10. Note that we have an isomorphism $\operatorname{Im}(\beta) \simeq \operatorname{Ker}(\gamma)$. The kernel of the map $\gamma: H_{\mathrm{syn}}^{2}\left(C_{F_{\infty}}, \mathcal{S}_{D}\right) \longrightarrow H_{\mathrm{syn}}^{2}\left(C_{F_{\infty}}, \mathcal{S}_{D}\right) \otimes \mathbb{Q}_{p}$ is $H_{\mathrm{syn}}^{2}\left(C_{F_{\infty}}, \mathcal{S}_{D}\right)(p)$. Recall that we have a short exact sequence:

$$
0 \longrightarrow \operatorname{Coker}\left(1-\varphi_{1}\right) \longrightarrow H_{\text {syn }}^{2}\left(C_{F_{\infty}}, \mathcal{S}_{D}\right) \longrightarrow \operatorname{Ker}\left(1-\varphi_{2}\right) \longrightarrow 0,
$$

By taking $p$-power torsion part of this sequence, we have

$$
0 \longrightarrow \operatorname{Coker}\left(1-\varphi_{1}\right)(p) \longrightarrow \operatorname{Im}(\beta) \longrightarrow \operatorname{Ker}\left(1-\varphi_{2}\right)(p) \text {. }
$$

Taking the Pontrjagin duals and then taking the $\Gamma^{\left(p^{\prime}\right)}$-coinvariants of the modules, we have the following:

$$
\left(\operatorname{Ker}\left(1-\varphi_{2}\right)(p)^{\vee}\right)_{\Gamma^{\left(p^{\prime}\right)}} \longrightarrow\left(\operatorname{Im}(\beta)^{\vee}\right)_{\Gamma^{\left(p^{\prime}\right)}} \longrightarrow\left(\operatorname{Coker}\left(1-\varphi_{1}\right)(p)^{\vee}\right)_{\Gamma^{\left(p^{\prime}\right)}} \longrightarrow 0,
$$

where the modules and the morphisms are defined over $\Lambda\left(\Gamma^{(p)}\right)$ as discussed around (7). By the sequence (9) and Lemma 2.5, it is enough to show that $\left(\operatorname{Coker}\left(1-\varphi_{1}\right)(p)^{\vee}\right)_{\Gamma\left(p^{\prime}\right)}$ and $\left(\operatorname{Ker}\left(1-\varphi_{2}\right)(p)^{\vee}\right)_{\Gamma^{\left(p^{\prime}\right)}}$ are finitely generated torsion $\Lambda\left(\Gamma^{(p)}\right)$-modules.

By the triangle (4), $\operatorname{Coker}\left(1-\varphi_{1}\right.$ ) (resp. $\operatorname{Ker}\left(1-\varphi_{2}\right)$ ) is a quotient (resp. submodule) of $H_{\text {crys }}^{1}\left(C_{F_{\infty}}^{\sharp} / W, D(-Z)\right)$ (resp. $\left.H_{\text {crys }}^{2}\left(C_{F_{\infty}}^{\sharp} / W, D^{0}(-Z)\right)\right)$. By Lemma 2.3, both $H_{\text {crys }}^{i}\left(C_{F_{\infty}}^{\sharp} / W, D^{0}(-Z)\right)$ and $H_{\text {crys }}^{i}\left(C_{F_{\infty}}^{\sharp} / W, D(-Z)\right)$ are finitely generated $W\left(\overline{\mathbb{F}}_{q}\right)$-modules. Hence, they are of the form $W\left(\overline{\mathbb{F}}_{q}\right)^{\oplus r_{0}} \oplus \bigoplus_{i=1}^{r} W_{n_{i}}\left(\overline{\mathbb{F}}_{q}\right)$, where the $n_{i} \geq 1$ are not necessarily distinct. Now, we will terminate the proof admitting the following claim, which will be shown below:

Claim 2.11. (1) Let $M$ be a $\Lambda(\Gamma)$-module such that $M \cong \oplus_{i=1}^{r} W_{n_{i}}\left(\overline{\mathbb{F}}_{q}\right)$, where the $n_{i} \geq 1$ are not necessarily distinct. Then

(a) For any $\Lambda(\Gamma)$-linear quotient $N$ of $M,\left(N^{\vee}\right)_{\Gamma^{\left(p^{\prime}\right)}}$ is a finitely generated torsion $\Lambda\left(\Gamma^{(p)}\right)$-module. 
(b) For any $\Lambda(\Gamma)$-submodule $N^{\prime}$ of $M,\left(N^{\prime \vee}\right)_{\Gamma^{\left(p^{\prime}\right)}}$ is a finitely generated torsion $\Lambda\left(\Gamma^{(p)}\right)$-module.

(2) For any $\Lambda(\Gamma)$-linear quotient $S$ of the $\Lambda(\Gamma)$-module $W\left(\overline{\mathbb{F}}_{q}\right), S(p)$ is equal to $S\left[p^{t}\right]$ for a sufficiently large integer $t$.

For $\operatorname{Ker}\left(1-\varphi_{2}\right)(p)^{\vee}$, we have the sequence:

$$
H_{\text {crys }}^{2}\left(C_{F_{\infty}}^{\sharp} / W, D^{0}(-Z)\right)(p)^{\vee} \longrightarrow \operatorname{Ker}\left(1-\varphi_{2}\right)(p)^{\vee} \longrightarrow 0 .
$$

Taking the $\Gamma^{\left(p^{\prime}\right)}$-coinvariant, we have:

$$
\left(H_{\text {crys }}^{2}\left(C_{F_{\infty}}^{\sharp} / W, D^{0}(-Z)\right)(p)^{\vee}\right)_{\Gamma^{\left(p^{\prime}\right)}} \longrightarrow\left(\operatorname{Ker}\left(1-\varphi_{2}\right)(p)^{\vee}\right)_{\Gamma^{\left(p^{\prime}\right)}} \longrightarrow 0 .
$$

Since $\left(H_{\text {crys }}^{2}\left(C_{F_{\infty}}^{\sharp} / W, D^{0}(-Z)\right)(p)^{\vee}\right)_{\Gamma^{\left(p^{\prime}\right)}}$ is a finitely generated torsion $\Lambda\left(\Gamma^{(p)}\right)$-module by Claim 2.11.1, $\left(\operatorname{Ker}\left(1-\varphi_{2}\right)(p)^{\vee}\right)_{\Gamma^{\left(p^{\prime}\right)}}$ is a finitely generated torsion $\Lambda\left(\Gamma^{(p)}\right)$-module by Lemma 2.5. For Coker $\left(1-\varphi_{1}\right)(p)^{\vee}$, we start from the following exact sequence of $\Lambda(\Gamma)$ modules:

$$
0 \longrightarrow \operatorname{Im}\left(1-\varphi_{1}\right) \longrightarrow H_{\text {crys }}^{1}\left(C_{F_{\infty}}^{\sharp} / W, D(-Z)\right) \longrightarrow \operatorname{Coker}\left(1-\varphi_{1}\right) \longrightarrow 0 .
$$

By Claim 2.11,2 and by the remark on the structure on $H_{\text {crys }}^{1}\left(C_{F_{\infty}}^{\sharp} / W, D(-Z)\right)$ given before, there is a sufficiently large $s$ such that $\operatorname{Im}\left(1-\varphi_{1}\right)(p)\left(\operatorname{resp} . H_{\text {crys }}^{1}\left(C_{F_{\infty}}^{\sharp} / W, D(-Z)\right)(p)\right.$, $\left.\operatorname{Coker}\left(1-\varphi_{1}\right)(p)\right)$ is equal to $\operatorname{Im}\left(1-\varphi_{1}\right)\left[p^{s}\right]\left(\right.$ resp. $H_{\text {crys }}^{1}\left(C_{F_{\infty}}^{\sharp} / W, D(-Z)\right)\left[p^{s}\right]$, Coker $(1-$ $\left.\left.\varphi_{1}\right)\left[p^{s}\right]\right)$. Hence, we have the following sequence:

$$
\left(\operatorname{Im}\left(1-\varphi_{1}\right) /\left(p^{s}\right) \operatorname{Im}\left(1-\varphi_{1}\right)\right)^{\vee} \stackrel{a}{\longrightarrow} \operatorname{Coker}\left(1-\varphi_{1}\right)(p)^{\vee} \stackrel{b}{\longrightarrow} H_{\text {crys }}^{1}\left(C_{F_{\infty}}^{\sharp} / W, D(-Z)\right)(p)^{\vee}
$$

As is explained above, the image of the map $b$ in (10) is of the form $N^{\vee}$ for a $\Lambda(\Gamma)$-linear quotient $N$ of $\oplus_{i=1}^{r} W_{n_{i}}\left(\overline{\mathbb{F}}_{q}\right)$. Since $\operatorname{Im}\left(1-\varphi_{1}\right) /\left(p^{s}\right) \operatorname{Im}\left(1-\varphi_{1}\right)$ is a $\Lambda(\Gamma)$-linear quotient of $H_{\text {crys }}^{1}\left(C_{F_{\infty}}^{\sharp} / W, D^{0}(-Z)\right) /\left(p^{s}\right) H_{\text {crys }}^{1}\left(C_{F_{\infty}}^{\sharp} / W, D^{0}(-Z)\right)$, the image of the map $a$ is of the form $N^{\prime \vee}$ for a $\Lambda(\Gamma)$-linear submodule $N^{\prime}$ of $\oplus_{j=1}^{r^{\prime}} W_{n_{j}^{\prime}}\left(\overline{\mathbb{F}}_{q}\right)$. Since $\Gamma^{\left(p^{\prime}\right)}$ is pro-cyclic group, the sequence (10) induces the following sequence:

$$
\left(N^{\prime \vee}\right)_{\Gamma^{\left(p^{\prime}\right)}} \longrightarrow\left(\operatorname{Coker}\left(1-\varphi_{1}\right)(p)^{\vee}\right)_{\Gamma^{\left(p^{\prime}\right)}} \longrightarrow\left(N^{\vee}\right)_{\Gamma^{\left(p^{\prime}\right)}} \longrightarrow 0 .
$$

Since the modules on the right and the left are finitely generated torsion $\Lambda\left(\Gamma^{(p)}\right)$-modules by Claim 2.11 , $1,\left(\operatorname{Coker}\left(1-\varphi_{1}\right)(p)^{\vee}\right)_{\Gamma^{\left(p^{\prime}\right)}}$ is a finitely generated torsion $\Lambda\left(\Gamma^{(p)}\right)$-module by Lemma 2.5.

To finish the proof of Lemma 2.10, we prove Claim 2.11. Since $W_{m}\left(\overline{\mathbb{F}}_{q}\right)$ is a successive extension of $W_{1}\left(\overline{\mathbb{F}}_{q}\right)=\overline{\mathbb{F}}_{q}$, it is enough to show the first assertion only for $M=W_{1}\left(\overline{\mathbb{F}}_{q}\right)=$ $\overline{\mathbb{F}}_{q}$. Let $U$ be an open subgroup of $\Gamma,\left(\overline{\mathbb{F}}_{q}\right)^{U}$ is a finite extension of $\mathbb{F}_{q}$. We have a natural $\Lambda(\Gamma)$-linear isomorphism $\left(\overline{\mathbb{F}}_{q}\right)^{U} \cong \mathbb{F}_{q}\left[(\Gamma / U)^{\vee}\right]$. If $\Gamma / U$ is sufficiently small so that the order of $\Gamma / U$ divides $q-1$, we can take a basis $\left\{x_{1}, \cdots, x_{l}\right\}$ of $\left(\overline{\mathbb{F}}_{q}\right)^{U}$ over $\mathbb{F}_{q}$, so that the action of $\Gamma$ on $\left(\overline{\mathbb{F}}_{q}\right)^{U}$ is represented by a diagonal matrix. For each member $x_{i}$ in the above set of basis, $g \mapsto\left(x_{i}\right)^{g} / x_{i}$ gives a character of $\Gamma$. Every character of $\Gamma$ factored by $\Gamma / U$ is given this way and for different $x_{i}, x_{j}$ the associated characters are different. This explains the canonical isomorphism $\left(\overline{\mathbb{F}}_{q}\right)^{U} \cong \mathbb{F}_{q}\left[(\Gamma / U)^{\vee}\right]$ for these special $U$ 's. Since they are rather elementary, we do not give further explanation nor the proof on the above isomorphisms. By taking an inductive limit of $\left(\overline{\mathbb{F}}_{q}\right)^{U} \cong \mathbb{F}_{q}\left[(\Gamma / U)^{\vee}\right]$ with respect to open subgroups $U$ of $\Gamma$, we have:

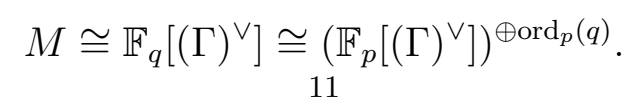


Hence, it suffices to show the first assertion only for a $\Lambda(\Gamma)$-linear quotient $N$ of $\mathbb{F}_{p}\left[(\Gamma)^{\vee}\right]$. Taking Pontrjagin dual, $N^{\vee}$ is a $\Lambda(\Gamma)$-submodule of $\left(\mathbb{F}_{p}\left[(\Gamma)^{\vee}\right]\right)^{\vee} \cong \mathbb{F}_{p}[[\Gamma]] \cong \Lambda(\Gamma) /(p)$. Hence we have $N^{\vee}=I$ for an ideal $I$ of $\mathbb{F}_{p}[[\Gamma]]$. Since $\Gamma^{\left(p^{\prime}\right)}$ is procyclic, the short exact sequence:

$$
0 \longrightarrow I \longrightarrow \mathbb{F}_{p}[[\Gamma]] \longrightarrow \mathbb{F}_{p}[[\Gamma]] / I \longrightarrow 0
$$

induces the following sequence:

$$
\left(\mathbb{F}_{p}[[\Gamma]] / I\right)^{\Gamma^{\left(p^{\prime}\right)}} \longrightarrow\left(N^{\vee}\right)_{\Gamma^{\left(p^{\prime}\right)}} \longrightarrow\left(\mathbb{F}_{p}[[\Gamma]]\right)_{\Gamma^{\left(p^{\prime}\right)}} \longrightarrow\left(\mathbb{F}_{p}[[\Gamma]] / I\right)_{\Gamma^{\left(p^{\prime}\right)}} \longrightarrow 0 .
$$

The third term $\left(\mathbb{F}_{p}[[\Gamma]]\right)_{\Gamma^{\left(p^{\prime}\right)}}$ is isomorphic to $\mathbb{F}_{p}\left[\left[\Gamma^{(p)}\right]\right] \cong \Lambda\left(\Gamma^{(p)}\right) /(p)$. For the first term, we decompose as $\mathbb{F}_{p}[[\Gamma]] / I \cong \mathbb{F}_{p}\left[\left[\Gamma^{(p)}\right]\right]\left[\left[\Gamma^{\left(p^{\prime}\right)}\right]\right] / I \cong\left(\left(\left(\mathbb{F}_{p}\left[\left[\Gamma^{(p)}\right]\right] / I_{0}\right)\right)\left[\left[\Gamma^{\left(p^{\prime}\right)}\right]\right]\right) / \bar{I}$ where $I_{0}=$ $I \cap \mathbb{F}_{p}\left[\left[\Gamma^{(p)}\right]\right]$ and $\bar{I}$ is the image of $I$ via $\mathbb{F}_{p}[[\Gamma]] \cong \mathbb{F}_{p}\left[\left[\Gamma^{(p)}\right]\right]\left[\left[\Gamma^{\left(p^{\prime}\right)}\right]\right] \rightarrow\left(\mathbb{F}_{p}\left[\left[\Gamma^{(p)}\right]\right] / I_{0}\right)\left[\left[\Gamma^{\left(p^{\prime}\right)}\right]\right]$. Now, it is easy to see that $\left(R\left[\left[\Gamma^{\left(p^{\prime}\right)}\right]\right] / \bar{I}\right)^{\Gamma^{\left(p^{\prime}\right)}}=R$ when $R$ is a pro- $p$ algebra invariant under $\Gamma^{\left(p^{\prime}\right)}$ and $\bar{I}$ is the ideal of $R\left[\left[\Gamma^{\left(p^{\prime}\right)}\right]\right]$ such that $R \cap \bar{I}=0$. Hence, the first term of (13) is isomorphic to $\mathbb{F}_{p}\left[\left[\Gamma^{(p)}\right]\right] / I_{0}$, which is a finitely generated torsion $\Lambda\left(\Gamma^{(p)}\right)$-module. Since $\Lambda\left(\Gamma^{(p)}\right)$ is noetherian, the assertion (a) is an immediate consequence of the sequence (13).

For the assertion (b), by the same argument as above, it is sufficient to prove when $N^{\prime}$ is a $\Lambda(\Gamma)$-linear submodule of $\mathbb{F}_{p}\left[(\Gamma)^{\vee}\right]$. Then we have a $\Lambda\left(\Gamma^{(p)}\right)$-linear map $\Lambda\left(\Gamma^{(p)}\right) /(p) \rightarrow$ $\left(N^{\prime \vee}\right)_{\Gamma^{\left(p^{\prime}\right)}}$. This completes the proof of (b).

By the similar argument as above, we have $W\left(\overline{\mathbb{F}}_{q}\right) \cong\left(\lim _{n}\left(\mathbb{Z} /\left(p^{n}\right)\left[(\Gamma)^{\vee}\right]\right)\right)^{\oplus \operatorname{ord}_{p}(q)}$. It is not difficult to show that only non-trivial $\Lambda(\Gamma)$-linear quotients of $\lim _{n}\left(\mathbb{Z} /\left(p^{n}\right)\left[(\Gamma)^{\vee}\right]\right)$ is either $\lim _{n}\left(\mathbb{Z} /\left(p^{n}\right)\left[(\Gamma)^{\vee}\right]\right)$ itself or $\mathbb{Z} /\left(p^{t}\right)\left[(\Gamma)^{\vee}\right]$ for some $t$. This suffices for the second assertion. This completes the proof of Claim 2.11 (and hence completes the proof of Lemma 2.10).

Before starting the proof of Theorem 1.7, we recall the following result:

Proposition 2.12. (1) Let $A / F$ an abelian variety satisfying the condition $(\mathbf{S S})$. Let $D(\mathcal{A})$ be the Dieudonné crystal over $C_{F_{\infty}^{(p)}}^{\sharp} / W$ associated to the Néron model $\mathcal{A}$ of $A \times_{F} F_{\infty}^{(p)}$. Then we have a monomorphism of $\Lambda\left(\Gamma^{(p)}\right)$-modules

$$
X\left(A / F_{\infty}^{(p)}\right) \longrightarrow X\left(D(\mathcal{A}) / F_{\infty}^{(p)}\right) .
$$

(2) Let $\mathcal{G}$ be a p-divisible group over $C_{F_{\infty}^{(p)}}$, then $X\left(D(\mathcal{G}) / F_{\infty}^{(p)}\right)$ is isomorphic to the Pontrjagin dual of $H_{\mathrm{fl}}^{1}\left(C_{F_{\infty}^{(p)}}, \mathcal{G}\right)$.

Proof. The first assertion is proved as in $[\mathrm{K}-\mathrm{T}, \S 2.5,5.13]$. The second assertion is reduced to [K-T, $\S 5.10]$.

Proof of Theorem 1.7. By Lemma 2.1, we can assume that $A / F$ has semi-stable reduction.

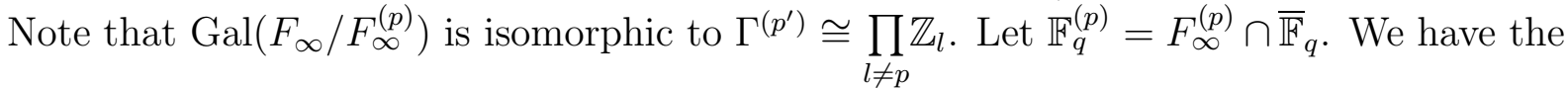
following commutative diagram:

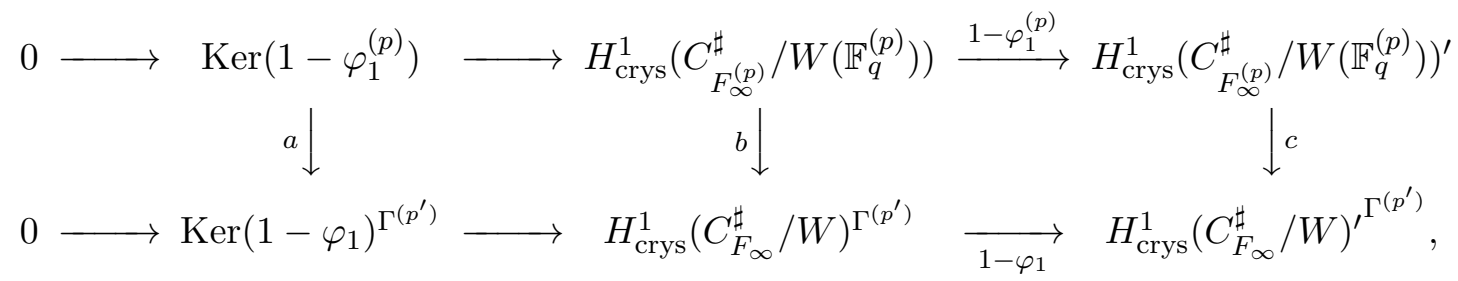


where $H_{\text {crys }}^{1}\left(C_{F_{\infty}^{(p)}} / W\left(\mathbb{F}_{q}^{(p)}\right)\right)\left(\right.$ resp. $\left.H_{\text {crys }}^{1}\left(C_{F_{\infty}^{(p)}} / W\left(\mathbb{F}_{q}^{(p)}\right)\right)^{\prime}\right)$ means $H_{\text {crys }}^{1}\left(C_{F_{\infty}^{(p)}}^{\sharp} / W\left(\mathbb{F}_{q}^{(p)}\right), D^{0}(-Z) \otimes\right.$ $\left.\mathbb{Q}_{p} / \mathbb{Z}_{p}\right)\left(\right.$ resp. $\left.H_{\text {crys }}^{1}\left(C_{F_{\infty}^{(p)}}^{\sharp} / W\left(\mathbb{F}_{q}^{(p)}\right), D(-Z) \otimes \mathbb{Q}_{p} / \mathbb{Z}_{p}\right)\right)$ and we take the similar definitions for $F_{\infty}$. We denote by $\varphi_{i}^{(p)}$ the frobenius operator over $F_{\infty}^{(p)}$. The vertical maps $b$ and $c$ in the diagram are isomorphism by crystalline base change theorem. Thus, the map $a$ is isomorphism. By the triangle (4), we also have the following diagram:

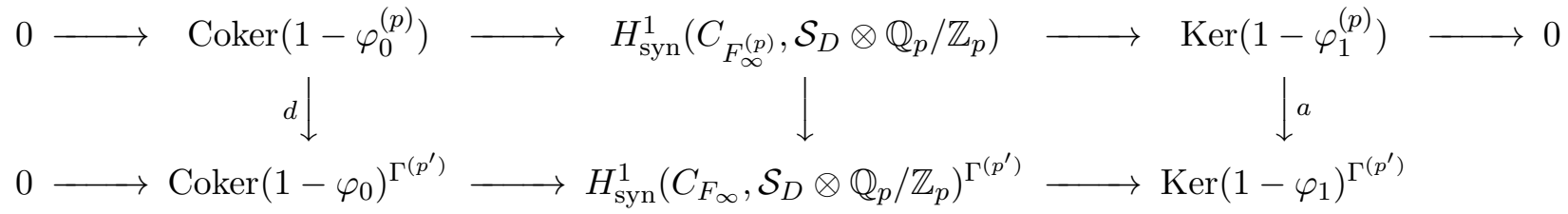

We have the following claim:

Claim 2.13. The Pontrjagin dual of the cokernel of the map:

$1-\varphi_{0}^{(p)}: H_{\text {crys }}^{0}\left(C_{F_{\infty}^{(p)}}^{\sharp} / W\left(\mathbb{F}_{q}^{(p)}\right), D^{0}(-Z) \otimes \mathbb{Q}_{p} / \mathbb{Z}_{p}\right) \longrightarrow H_{\text {crys }}^{0}\left(C_{F_{\infty}^{(p)}}^{\sharp} / W\left(\mathbb{F}_{q}^{(p)}\right), D(-Z) \otimes \mathbb{Q}_{p} / \mathbb{Z}_{p}\right)$

is a finitely generated torsion $\Lambda\left(\Gamma^{(p)}\right)$-module.

By this claim, the Pontrjagin dual of Coker $\left(1-\varphi_{0}^{(p)}\right)$ is a finitely generated torsion $\Lambda\left(\Gamma^{(p)}\right)$-module. Taking the Pontrjagin dual of the above commutative diagram, we have a $\Lambda\left(\Gamma^{(p)}\right)$-linear map $X\left(D(\mathcal{A}) / F_{\infty}\right)_{\Gamma\left(p^{\prime}\right)} \longrightarrow X\left(D(\mathcal{A}) / F_{\infty}^{(p)}\right)$ whose cokernel is a torsion $\Lambda\left(\Gamma^{(p)}\right)$-module of finite type. On the other hand, $X\left(D(\mathcal{A}) / F_{\infty}\right)_{\Gamma^{\left(p^{\prime}\right)}}$ is a finitely generated torsion $\Lambda\left(\Gamma^{(p)}\right)$-module by Proposition 2.6. Finally we will prove Claim 2.13, This will complete the proof of Theorem [1.7 by Proposition 2.12, We consider the following commutative diagram whose vertical maps are all $1-\varphi_{i}^{(p)}$ :

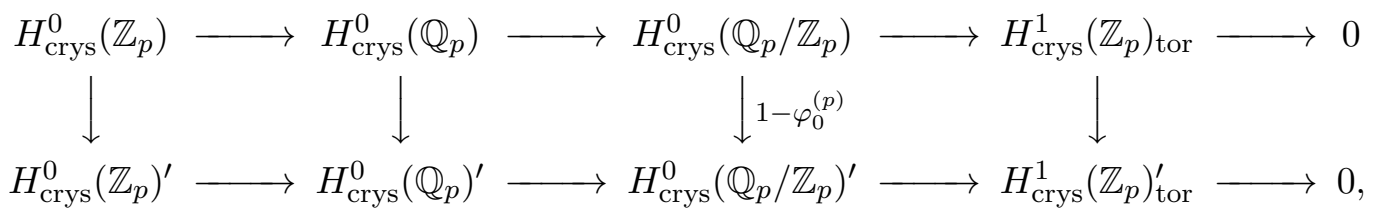

where $H_{\text {crys }}^{i}(*)$ in the upper line with $*=\mathbb{Z}_{p}, \mathbb{Q}_{p}, \mathbb{Q}_{p} / \mathbb{Z}_{p}$ means $H_{\text {crys }}^{i}\left(C_{F_{\infty}^{(p)}}^{\sharp} / W\left(\mathbb{F}_{q}^{(p)}\right), D^{0}(-Z) \otimes\right.$ $*), H_{\text {crys }}^{i}(*)^{\prime}$ in the lower line means $H_{\text {crys }}^{i}\left(C_{F_{\infty}^{(p)}}^{\sharp} / W\left(\mathbb{F}_{q}^{(p)}\right), D(-Z) \otimes *\right)$ and ()$_{\text {tor }}$ is the $\mathbb{Z}_{p^{-}}$ torsion part. We can show as in Lemma 2.3 (a) that the map $1: H_{\text {crys }}^{0}\left(\mathbb{Q}_{p}\right) \longrightarrow H_{\text {crys }}^{0}\left(\mathbb{Q}_{p}\right)^{\prime}$ induces a natural isomorphism of finite dimensional $W\left(\mathbb{F}_{q}^{(p)}\right) \otimes \mathbb{Q}_{p}$-vector spaces. Since $\varphi_{0}^{(p)}$ is $\sigma$-linear, $1-\varphi_{0}^{(p)}: H_{\text {crys }}^{0}\left(\mathbb{Q}_{p}\right) \longrightarrow H_{\text {crys }}^{0}\left(\mathbb{Q}_{p}\right)^{\prime}$ is surjective (see [EL, Lemma 6.2] for a similar argument). By the diagram (14) and by the snake lemma, Coker[1 $\left.\varphi_{0}^{(p)}: H_{\text {crys }}^{0}\left(\mathbb{Q}_{p} / \mathbb{Z}_{p}\right) \rightarrow H_{\text {crys }}^{0}\left(\mathbb{Q}_{p} / \mathbb{Z}_{p}\right)^{\prime}\right]$ is isomorphic to Coker $\left[1-\varphi_{1}^{(p)}: H_{\text {crys }}^{1}\left(\mathbb{Z}_{p}\right)_{\text {tor }} \rightarrow\right.$ $\left.H_{\text {crys }}^{1}\left(\mathbb{Z}_{p}\right)_{\text {tor }}^{\prime}\right]$. Thus, Coker $\left[1-\varphi_{0}^{(p)}: H_{\text {crys }}^{0}\left(\mathbb{Q}_{p} / \mathbb{Z}_{p}\right) \rightarrow H_{\text {crys }}^{0}\left(\mathbb{Q}_{p} / \mathbb{Z}_{p}\right)^{\prime}\right]$ is a $\Lambda\left(\Gamma^{(p)}\right)$-linear quotient of a finitely generated torsion $W\left(\mathbb{F}_{q}^{(p)}\right)$-module $H_{\text {crys }}^{1}\left(\mathbb{Z}_{p}\right)_{\text {tor }}^{\prime}$. This completes the proof of Claim 2.13,

\section{3. $\mu$-INVARIANTS OF $X\left(A / F_{\infty}^{(p)}\right)$}

We showed that $X\left(A / F_{\infty}^{(p)}\right)$ is a torsion $\Lambda\left(\Gamma^{(p)}\right)$-module for an abelian variety $A$ over $F$. In this section, we will prove that the $\mu$-invariant of $X\left(A / F_{\infty}^{(p)}\right)$ is zero under certain assumptions (Theorem 1.8). 
Before giving the proof, we prepare several fundamental facts which will be necessary in the proof.

Lemma 3.1. Let $K$ be a finitely generated one-variable function field over a field $\mathbb{F}$ contained in $\overline{\mathbb{F}}_{q}$ and let $A$ be an abelian variety over $K$. Then, the $\operatorname{Selmer}$ group $\operatorname{Sel}(A / K)$ is isomorphic to the flat cohomology $H_{\mathrm{fl}}^{1}\left(C_{K}, \mathcal{A}\{p\}\right)$ if $A$ has everywhere good reduction over $K$, where $\mathcal{A}$ is the model of $A$ over $C_{K}$. Here, $C_{K}$ denotes the projective smooth curve which is the model of a function field $K$.

Let us recall the following claim:

Claim 3.2. Let $V$ be a smooth curve over a finite field $\mathbb{F}_{q}$ and let $U \subset V$ be a dense open subset. For any finite flat group scheme $\mathcal{F}$ over $\mathcal{O}_{V}$, we have the following natural exact sequence:

$$
0 \longrightarrow H_{\mathrm{fl}}^{1}(V, \mathcal{F}) \longrightarrow H_{\mathrm{fl}}^{1}(U, \mathcal{F}) \longrightarrow \bigoplus_{v \in V \backslash U} \frac{H_{\mathrm{fl}}^{1}\left(K_{v}, F\right)}{H_{\mathrm{fl}}^{1}\left(\mathcal{O}_{v}, \mathcal{F}\right)},
$$

where $\mathcal{O}_{v}$ is the completion of $\mathcal{O}_{V}$ at the closed point $v \in V, K_{v}$ is the field of fraction of $\mathcal{O}_{v}$ and $F$ is the generic fiber of $F$.

Though this claim seems to be known to the experts, we will give a short sketch of the proof by recalling fundamental facts on flat cohomologies. For fundamental tools and facts used in the proof, the reader can refer to [Mi2, Chapter III].

Proof of Claim 3.2. Let $Z=V \backslash U$ be a closed subscheme of $V$. We have a usual localization sequence:

$$
H_{Z}^{1}(V, F) \longrightarrow H_{\mathrm{fl}}^{1}(V, \mathcal{F}) \longrightarrow H_{\mathrm{fl}}^{1}(U, \mathcal{F}) \longrightarrow H_{Z}^{2}(V, \mathcal{F})
$$

where $H_{Z}^{i}(V, \mathcal{F})$ is a flat cohomology with support in $Z$. By [Mi2, III, §7], we have:

$$
\left\{\begin{array}{l}
H_{Z}^{1}(V, \mathcal{F})=0 \\
H_{Z}^{2}(V, \mathcal{F})=\bigoplus_{v \in V \backslash U} H_{v}^{2}(V, \mathcal{F}) \cong \bigoplus_{v \in V \backslash U} \frac{H_{\mathrm{fl}}^{1}\left(K_{v}, F\right)}{H_{\mathrm{fl}}^{1}\left(\mathcal{O}_{v}, \mathcal{F}\right)}
\end{array}\right.
$$

This completes the proof.

Proof of Lemma 3.1. By Claim 3.2, we have:

$$
0 \longrightarrow H_{\mathrm{fl}}^{1}\left(C_{K}, \mathcal{A}\left[p^{m}\right]\right) \longrightarrow H_{\mathrm{fl}}^{1}\left(C_{K} \backslash Z, \mathcal{A}\left[p^{m}\right]\right) \longrightarrow \underset{v \in Z}{\oplus} \frac{H_{\mathrm{fl}}^{1}\left(K_{v}, A\left[p^{m}\right]\right)}{H_{\mathrm{fl}}^{1}\left(\mathcal{O}_{v}, \mathcal{A}\left[p^{m}\right]\right)}
$$

By taking inductive limit with respect to $Z$ and $m$, we have:

$$
0 \longrightarrow H_{\mathrm{fl}}^{1}\left(C_{K}, \mathcal{A}\{p\}\right) \longrightarrow H_{\mathrm{fl}}^{1}(K, A\{p\}) \longrightarrow \underset{v \in C_{K}}{\oplus} \frac{H_{\mathrm{f}}^{1}\left(K_{v}, A\{p\}\right)}{H_{\mathrm{fl}}^{1}\left(\mathcal{O}_{v}, \mathcal{A}\{p\}\right)}
$$

On the other hand, we easily show that $\frac{H_{\mathrm{fl}}^{1}\left(K_{v}, A\{p\}\right)}{H_{\mathrm{f}}^{1}\left(\mathcal{O}_{v}, \mathcal{A}\{p\}\right)} \cong H^{1}\left(K_{v}, A\right)(p)$ since $H_{\mathrm{fl}}^{1}\left(\mathcal{O}_{v}, \mathcal{A}\right) \cong$ $H_{\mathrm{fl}}^{1}\left(\mathbb{F}_{v}, \overline{\mathcal{A}}_{v}\right)=0$ by $\left[\mathrm{L}\right.$, where $\mathbb{F}_{v}$ is the residue field of $\mathcal{O}_{v}$ and $\overline{\mathcal{A}}_{v}$ is the special fiber of $\mathcal{A}$ at $\operatorname{Spec}\left(\mathbb{F}_{v}\right)$. This completes the proof of Lemma 3.1 by comparing with the exact sequence (11) which defines the Selmer group.

By using the following lemma, we reduce the proof of Theorem 1.8 into a simplified situation . 
Lemma 3.3. Let $F^{\prime} / F$ be a finite Galois extension. We denote by $A^{\prime}$ the extension $A^{\prime}=A \times_{F} F^{\prime}$. If $\mu\left(X\left(A^{\prime} / F_{\infty}^{\prime,(p)}\right)\right)=0$, then we have $\mu\left(X\left(A / F_{\infty}^{(p)}\right)\right)=0$.

Proof. The assertion that $\mu\left(X\left(A / F_{\infty}^{(p)}\right)\right)=0$ (resp. $\left.\mu\left(X\left(A^{\prime} / F_{\infty}^{\prime,(p)}\right)\right)=0\right)$ is equivalent to the assertion that $X\left(A / F_{\infty}^{(p)}\right)$ (resp. $X\left(A^{\prime} / F_{\infty}^{\prime(p)}\right)$ ) is a finitely generated $\mathbb{Z}_{p}$-module. As the proof of Lemma 2.1, we have a natural homomorphism $X\left(A^{\prime} / F_{\infty}^{\prime(p)}\right)_{J} \longrightarrow X\left(A / F_{\infty}^{(p)}\right)$ whose cokernel is finite (Here, $J$ is $\operatorname{Gal}\left(F_{\infty}^{\prime(p)} / F_{\infty}^{(p)}\right)$ ). Hence we complete the proof.

By Lemma 3.3, we may (and we will) assume that $A / F$ is isomorphic to an ordinary abelian variety or a supersingular abelian variety defined over a finite field in order to prove Theorem 1.8. First, we consider the case where the following condition is satisfied through this section for the proof of Theorem 1.8:

(OF) $A / F$ is isomorphic to an ordinary abelian variety defined over a finite field $\mathbb{F}_{q} \subset F$.

Note that $F_{\infty}$ is a Galois extension of $F_{\infty}^{(p)}$ with Galois group $\Gamma^{\left(p^{\prime}\right)} \cong \prod_{l \neq p} \mathbb{Z}_{l}$. As in the argument of the proof of Theorem 1.7, we have:

$$
H_{\mathrm{fl}}^{1}\left(C_{F_{\infty}^{(p)}}, A\{p\}\right)^{\vee} \cong\left(H_{\mathrm{fl}}^{1}\left(C_{F_{\infty}}, A\{p\}\right)^{\vee}\right)_{\Gamma^{\left(p^{\prime}\right)}}
$$

Thus we have reduced "finiteness" over $F_{\infty}^{(p)}$ to Lemma 3.4 ("finiteness" over $F_{\infty}$ ) below.

Lemma 3.4. Let us assume the condition ( $\mathrm{OF})$ for $A / F$. Then, $H_{\mathrm{f}}^{1}\left(C_{F_{\infty}}, A\{p\}\right)^{\vee}$ is a finitely generated $\mathbb{Z}_{p}$-modules.

Proof. Since $A$ is an abelian variety with good ordinary reduction, the connected part (resp. étale part) $A\{p\}^{\text {conn }}$ (resp. $A\{p\}^{\text {ét }}$ ) of $A\{p\}$ has rank $g=\operatorname{dim}(A)$. Note that a finite flat group scheme $A\{p\}^{\text {conn }}$ (resp. $A\{p\}^{\text {ét) }}$ over $C_{F_{\infty}}$ is a constant scheme defined over the base $\overline{\mathbb{F}}_{p}$ of the curve $C_{F_{\infty}}$. Since $A\{p\}^{\text {conn }}$ (resp. $A\{p\}^{\text {ét }}$ ) is isomorphic to $\left(\mu_{p^{\infty}}\right)^{g}$ (resp. $\left.\left(\mathbb{Q}_{p} / \mathbb{Z}_{p}\right)^{g}\right)$ over $\overline{\mathbb{F}}_{p}$, we have the following exact sequence:

$$
H_{\mathrm{fl}}^{1}\left(C_{F_{\infty}},\left(\mu_{p^{\infty}}\right)^{g}\right) \longrightarrow H_{\mathrm{fl}}^{1}\left(C_{F_{\infty}}, A\{p\}\right) \longrightarrow H_{\mathrm{fl}}^{1}\left(C_{F_{\infty}},\left(\mathbb{Q}_{p} / \mathbb{Z}_{p}\right)^{g}\right) .
$$

Thus we reduce the proof of Lemma 3.4 to the following claim:

Claim 3.5. $H_{\mathrm{fl}}^{1}\left(C_{F_{\infty}}, \mu_{p^{\infty}}\right)^{\vee}$ and $H_{\mathrm{fl}}^{1}\left(C_{F_{\infty}}, \mathbb{Q}_{p} / \mathbb{Z}_{p}\right)^{\vee}$ are free $\mathbb{Z}_{p}$-modules of finite rank.

Let us prove the claim in the rest of the proof. We first study $H_{\mathrm{fl}}^{1}\left(C_{F_{\infty}}, \mu_{p^{\infty}}\right)^{\vee}$. For a scheme $V$, [Mi1, Proposition 3.7] gives us an equality $H_{\mathrm{fl}}^{1}\left(V, \mathcal{O}_{V}^{\times}\right) \cong \operatorname{Pic}(V)$. On the other hand, we have an exact sequence:

$$
0 \longrightarrow \mu_{p^{n}} \longrightarrow \mathcal{O}_{V}^{\times} \stackrel{\times p^{n}}{\longrightarrow} \mathcal{O}_{V}^{\times} \longrightarrow 0
$$

on the flat site over $V$. This implies that $H_{\mathrm{fl}}^{1}\left(\operatorname{Spec}(R), \mu_{p^{n}}\right)=R^{\times} /\left(R^{\times}\right)^{p^{n}}$ for a local ring $R$. By Lemma 3.2, we have an exact sequence:

$$
\left.0 \longrightarrow H_{\mathrm{fl}}^{1}\left(C_{F_{\infty}}, \mu_{p^{n}}\right) \longrightarrow\left(F_{\infty}\right)^{\times} /\left(F_{\infty}\right)^{\times}\right)^{p^{n}} \longrightarrow \bigoplus_{v \in C_{F_{\infty}} \backslash \operatorname{Spec}\left(F_{\infty}\right)} \frac{\left(F_{\infty, v}\right)^{\times} /\left(\left(F_{\infty, v}\right)^{\times}\right)^{p^{n}}}{\mathcal{O}_{v}^{\times} /\left(\mathcal{O}_{v}^{\times}\right)^{p^{n}}}
$$

where $\mathcal{O}$ is the ring of integers of $F_{\infty}$. Note that we have:

$$
\frac{\left(F_{\infty, v}\right)^{\times} /\left(\left(F_{\infty, v}\right)^{\times}\right)^{p^{n}}}{\mathcal{O}_{v}^{\times} /\left(\mathcal{O}_{v}^{\times}\right)^{p^{n}}} \cong \frac{\left(F_{\infty, v}\right)^{\times} / \mathcal{O}_{v}^{\times}}{\left(\left(F_{\infty, v}\right)^{\times} / \mathcal{O}_{v}^{\times}\right)^{p^{n}}} \cong \mathbb{Z} /\left(p^{n}\right) .
$$


Let us consider the following commutative diagram:

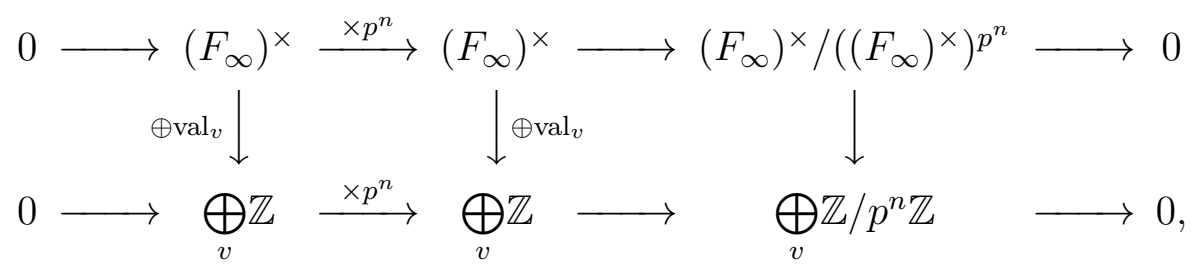

where $v$ runs the primes of $v \in C_{F_{\infty}} \backslash \operatorname{Spec}\left(F_{\infty}\right)$ for the summations in the lower line. The kernel of the middle vertical map is equal to $\left(\overline{\mathbb{F}}_{p}\right)^{\times}$. By applying the snake lemma to the above diagram, we get the following exact sequence

$$
0 \longrightarrow\left(\overline{\mathbb{F}}_{p}\right)^{\times} /\left(\left(\overline{\mathbb{F}}_{p}\right)^{\times}\right)^{p^{n}} \longrightarrow H_{\mathrm{fl}}^{1}\left(C_{F_{\infty}}, \mu_{p^{n}}\right) \rightarrow \mathrm{Cl}\left(F_{\infty}\right)\left[p^{n}\right] \longrightarrow 0 .
$$

Since $\left(\overline{\mathbb{F}}_{p}\right)^{\times} /\left(\left(\overline{\mathbb{F}}_{p}\right)^{\times}\right)^{p^{n}}$ is trivial, we have $H_{\mathrm{fl}}^{1}\left(C_{F_{\infty}}, \mu_{p^{\infty}}\right) \cong \mathrm{Cl}\left(F_{\infty}\right)\{p\}$. By [W], we have an isomorphism

$$
\mathrm{Cl}\left(F_{\infty}\right)\{p\} \simeq J_{F_{\infty}}\left(\overline{\mathbb{F}}_{p}\right)\{p\}
$$

where $J_{F_{\infty}}$ is the Jacobian variety associated to $C_{F_{\infty}}$. However, by $[\mathrm{Mu}]$, we have $J_{F_{\infty}}\left(\overline{\mathbb{F}}_{p}\right)\{p\} \simeq\left(\mathbb{Q}_{p} / \mathbb{Z}_{p}\right)^{r}$ for some $0 \leq r \leq \operatorname{genus}\left(C_{F_{\infty}}\right)$.

Next, we study $H_{\mathrm{fl}}^{1}\left(C_{F_{\infty}}, \mathbb{Q}_{p} / \mathbb{Z}_{p}\right)^{\vee}$. Let us recall the following exact sequence:

$$
H_{\mathrm{f}}^{i}\left(C_{F_{\infty}}, \mathbb{Z} /(p) \mathbb{Z}\right) \cong \operatorname{Ker}\left[H_{\mathrm{fl}}^{i}\left(C_{F_{\infty}}, \mathcal{O}_{C_{F_{\infty}}}\right) \stackrel{x \rightarrow x-x^{p}}{\longrightarrow} H_{\mathrm{ff}}^{i}\left(C_{F_{\infty}}, \mathcal{O}_{C_{F_{\infty}}}\right)\right],
$$

where the last map is induced by the Artin-Scherier sequence:

$$
0 \longrightarrow \mathbb{Z} /(p) \mathbb{Z} \longrightarrow \mathcal{O}_{C_{F_{\infty}}} \stackrel{x \rightarrow x-x^{p}}{\longrightarrow} \mathcal{O}_{C_{F_{\infty}}} \longrightarrow 0,
$$

on the flat site over $C_{F_{\infty}}$. Since $H_{\mathrm{fl}}^{i-1}\left(C_{F_{\infty}}, \mathcal{O}_{C_{F_{\infty}}}\right)$ is isomorphic to $H_{\mathrm{Zar}}^{i-1}\left(C_{F_{\infty}}, \mathcal{O}_{C_{F_{\infty}}}\right)$, it is a finite dimensional $\overline{\mathbb{F}}_{q}$-vector space. Hence $H_{\mathrm{fl}}^{i}\left(C_{F_{\infty}}, \mathcal{O}_{C_{F_{\infty}}}\right) \stackrel{x \rightarrow x-x^{p}}{\longrightarrow} H_{\mathrm{fl}}^{i}\left(C_{F_{\infty}}, \mathcal{O}_{C_{F_{\infty}}}\right)$ is surjective for any $i$. Further, $H_{\mathrm{Zar}}^{1}\left(C_{F_{\infty}}, \mathcal{O}_{C_{F_{\infty}}}\right)$ is isomorphic to an $r$-dimensional vector space over $\overline{\mathbb{F}}_{q}$. We have $H_{\mathrm{fl}}^{1}\left(C_{F_{\infty}}, \mathbb{Z} /(p) \mathbb{Z}\right) \cong(\mathbb{Z} /(p) \mathbb{Z})^{\oplus r}$ by (16). On the other hand, $H_{\mathrm{f}}^{2}\left(C_{F_{\infty}}, \mathbb{Z} /(p) \mathbb{Z}\right)$ is trivial since $H_{\mathrm{ff}}^{2}\left(C_{F_{\infty}}, \mathcal{O}_{C_{F_{\infty}}}\right)=H_{\mathrm{Zar}}^{2}\left(C_{F_{\infty}}, \mathcal{O}_{C_{F_{\infty}}}\right)$ is trivial. This gives the following exact sequence:

$$
0 \longrightarrow H_{\mathrm{fl}}^{1}\left(C_{F_{\infty}}, \mathbb{Z} /(p) \mathbb{Z}\right) \longrightarrow H_{\mathrm{fl}}^{1}\left(C_{F_{\infty}}, \mathbb{Q}_{p} / \mathbb{Z}_{p}\right) \stackrel{\times p}{\longrightarrow} H_{\mathrm{fl}}^{1}\left(C_{F_{\infty}}, \mathbb{Q}_{p} / \mathbb{Z}_{p}\right) \longrightarrow 0
$$

Since $H_{\mathrm{f}}^{1}\left(C_{F_{\infty}}, \mathbb{Q}_{p} / \mathbb{Z}_{p}\right)^{\vee} /(p) H_{\mathrm{fl}}^{1}\left(C_{F_{\infty}}, \mathbb{Q}_{p} / \mathbb{Z}_{p}\right)^{\vee}$ is finite, $H_{\mathrm{fl}}^{1}\left(C_{F_{\infty}}, \mathbb{Q}_{p} / \mathbb{Z}_{p}\right)^{\vee}$ is a finitely generated $\mathbb{Z}_{p}$-module by Nakayama's lemma. Further, $H_{\mathrm{ff}}^{1}\left(C_{F_{\infty}}, \mathbb{Q}_{p} / \mathbb{Z}_{p}\right)^{v}$ has no non-trivial $p$-torsion elements since $H_{\mathrm{fl}}^{1}\left(C_{F_{\infty}}, \mathbb{Q}_{p} / \mathbb{Z}_{p}\right)$ is $p$-divisible. Thus, $H_{\mathrm{fl}}^{1}\left(C_{F_{\infty}}, \mathbb{Q}_{p} / \mathbb{Z}_{p}\right)^{\vee}$ is a free $\mathbb{Z}_{p}$-module of rank $r$. This completes the proof of the claim and hence the proof of Lemma 3.4 is completed.

By a remark given before Lemma 3.4, the proof of Theorem 1.8 has been done in the ordinary case.

Next, we discuss the supersingular case. By Lemma 3.3, we may assume the case where the following condition is satisfied:

(SF) $A / F$ is isomorphic to a supersingular abelian variety defined over a finite field $\mathbb{F}_{q} \subset F$ and the proper smooth curve $C_{F}$ which is the model of $F$ has invertible HasseWitt matrix.

The assertion that $\mu\left(X\left(A / F_{\infty}\right)\right)=0$ is equivalent to the assertion that $H_{\mathrm{fl}}^{1}\left(C_{F_{\infty}}, A\{p\}\right)$ is 
p-divisible. Now, we consider the following exact sequence:

$$
H_{\mathrm{fl}}^{1}\left(C_{F_{\infty}}, A\{p\}\right) \stackrel{\times p}{\longrightarrow} H_{\mathrm{fl}}^{1}\left(C_{F_{\infty}}, A\{p\}\right) \longrightarrow H_{\mathrm{fl}}^{2}\left(C_{F_{\infty}}, A[p]\right) .
$$

Let $\boldsymbol{\alpha}_{p}$ be a finite group scheme defined to be the kernel of the frobenius map $F$ : $\mathbb{G}_{a} \longrightarrow \mathbb{G}_{a}$. Since $A$ is supersingular, $A[p]$ is isomorphic to $\boldsymbol{\alpha}_{p}^{\oplus 2 g}$, it suffices to show that $H^{2}\left(C_{F_{\infty}}, \boldsymbol{\alpha}_{p}\right)=0$. Now, from the exact sequence

$$
H_{\mathrm{fl}}^{1}\left(C_{F_{\infty}}, \mathcal{O}_{C_{F_{\infty}}}\right) \stackrel{F}{\longrightarrow} H_{\mathrm{fl}}^{1}\left(C_{F_{\infty}}, \mathcal{O}_{C_{F_{\infty}}}\right) \longrightarrow H_{\mathrm{fl}}^{2}\left(C_{F_{\infty}}, \boldsymbol{\alpha}_{p}\right) \longrightarrow 0,
$$

the assertion that $H^{2}\left(C_{F_{\infty}}, \boldsymbol{\alpha}_{p}\right)=0$ is equivalent to the assertion that the map $F$ on $H_{\mathrm{fl}}^{1}\left(C_{F_{\infty}}, \mathcal{O}_{C_{F_{\infty}}}\right)$ is surjective. Since the last assertion is equivalent to the assumption that the Hasse-Witt matrix for $C_{F}$ is invertible, we complete the proof for the first assertion of Theorem 1.8 .

If the Hasse-Witt matrix for $C_{F}$ is not invertible, the kernel of $F$ on $H_{\mathrm{fl}}^{1}\left(C_{F_{\infty}}, \mathcal{O}_{C_{F_{\infty}}}\right)$ contains a non-trivial $\overline{\mathbb{F}}_{p}$-vector space and the Frobenius on $H_{\mathrm{fl}}^{0}\left(C_{F_{\infty}}, \mathcal{O}_{C_{F_{\infty}}}\right) \cong \overline{\mathbb{F}}_{p}$ is surjective. Thus $H_{\mathrm{f}}^{1}\left(C_{F_{\infty}}, A[p]\right)^{\vee} \cong X\left(A / F_{\infty}^{(p)}\right) /(p) X\left(A / F_{\infty}^{(p)}\right)$ has infinite $p$-rank and $\mu\left(X\left(A / F_{\infty}^{(p)}\right)\right)$ has to be positive if the Hasse-Witt matrix for $C_{F}$ is not invertible. This completes the proof of Theorem 1.8 .

\section{A result over a $p$-ADic Lie extension $L$}

In this section, we prove Theorem 1.9, For the proof of Theorem 1.9, we introduce following new Selmer group:

Definition 4.1. Let $A$ be an abelian variety over $F$ and let $S$ be a finite set of primes of $F$. By using the extension $0 \longrightarrow A\{p\}^{\text {conn }} \longrightarrow A\{p\} \longrightarrow A\{p\}^{\text {ét }} \longrightarrow 0$ of p-divisible groups over $F$, we define the Selmer group over an algebraic extension $K$ over $F$ as follows:

$$
\operatorname{Sel}^{S}(A / K):=\operatorname{Ker}\left[H_{\mathrm{fl}}^{1}(K, A\{p\}) \longrightarrow \prod_{v \in S_{K}} H_{\mathrm{fl}}^{1}\left(K_{v}, A\{p\}^{e ́ t}\right) \times \prod_{v \notin S_{K}} H_{\mathrm{fl}}^{1}\left(K_{v}, A\right)\right]
$$

where $S_{K}$ is a set of primes of $K$ lying over the primes in $S$.

From now on, we fix a $g$-dimensional abelian variety $A$ over $F$, which has good reduction outside a finite set $S$ of primes in $F$ and has ordinary reduction at each prime in $S$ as in Theorem 1.9. We give the following lemma on comparison between Selmer groups:

Lemma 4.2. Let the assumption be as in Theorem 1.9.

(1) For any extension $K$ of $F, \operatorname{Sel}(A / K)$ is naturally identified as a subgroup of $\operatorname{Sel}^{S}(A / K)$.

(2) For any $K$ contained in $F_{\infty}$, the quotient $\operatorname{Sel}^{S}(A / K) / \operatorname{Sel}(A / K)$ is a cofinitely generated $\mathbb{Z}_{p}$-module whose corank is less than or equal to $g \sharp S$.

Proof. For an extension $K$ of $F$, we denote by $B_{K_{v}}$ the subgroup of $H_{\mathrm{fl}}^{1}\left(K_{v}, A\{p\}\right)$ given by

$B_{K_{v}}:=\operatorname{Ker}\left[H_{\mathrm{fl}}^{1}\left(K_{v}, A\{p\}\right) \longrightarrow H_{\mathrm{fl}}^{1}\left(K_{v}, A\{p\}^{\mathrm{ét}}\right)\right]=\operatorname{Im}\left[H_{\mathrm{fl}}^{1}\left(K_{v}, A\{p\}^{\mathrm{conn}}\right) \longrightarrow H_{\mathrm{fl}}^{1}\left(K_{v}, A\{p\}\right)\right]$.

Recall that $A\left(K_{v}\right) \otimes \mathbb{Q}_{p} / \mathbb{Z}_{p}$ is regarded as a subgroup of $H_{\mathrm{fl}}^{1}\left(K_{v}, A\{p\}\right)$ and the kernel of $H_{\mathrm{fl}}^{1}\left(K_{v}, A\{p\}\right) \longrightarrow H_{\mathrm{fl}}^{1}\left(K_{v}, A\right)$ is equal to $A\left(K_{v}\right) \otimes \mathbb{Q}_{p} / \mathbb{Z}_{p}$. By comparing the definition of $\operatorname{Sel}(A / K)$ and $\operatorname{Sel}^{S}(A / K)$ (11) and (17), respectively), $\operatorname{Sel}(A / K)$ is a subgroup of 
$\operatorname{Sel}^{S}(A / K)$ if and only if $A\left(K_{v}\right) \otimes \mathbb{Q}_{p} / \mathbb{Z}_{p}$ is contained in $B_{K_{v}}$ for every $v \in S$. We denote by $\mathcal{A}_{v}$ the Neron model on the ring of integers of $K_{v}$ associated to the abelian variety $A / K_{v}$. Let $\widehat{\mathcal{A}}_{v}$ be the formal completion of $\mathcal{A}_{v}$ along the zero section of the special fiber of $\mathcal{A}_{v}$. Since $A$ has ordinary reduction at $v, \widehat{\mathcal{A}}_{v}\left[p^{n}\right]$ is a finite flat group scheme which is étale locally isomorphic to a finite flat group scheme $\left(\mu_{p^{n}}\right)^{g \sharp S}$ over $\mathcal{O}_{v}$ whose generic fiber is isomorphic to the connected part $A\left[p^{n}\right]^{\text {conn }}$ of the finite flat group scheme $A\left[p^{n}\right]$ over $K_{v}$. We have the following diagram:

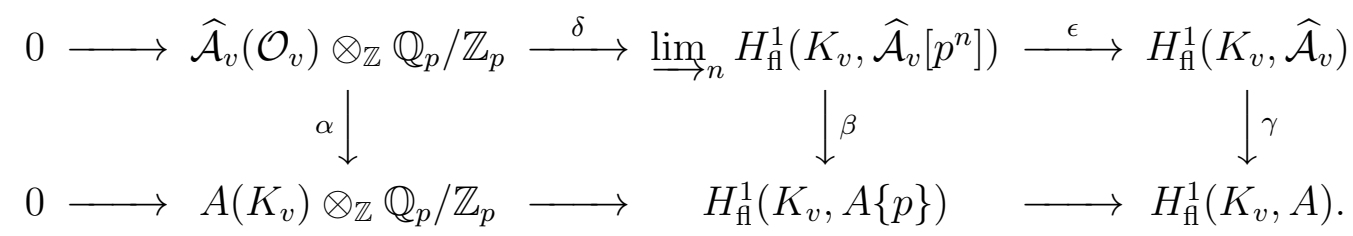

Since $\mathcal{A}_{v}\left(\mathcal{O}_{v}\right)=A\left(K_{v}\right)$, we have an exact sequence:

$$
0 \longrightarrow \widehat{\mathcal{A}}_{v}\left(\mathcal{O}_{v}\right) \longrightarrow A\left(K_{v}\right) \longrightarrow \overline{\mathcal{A}}_{v}\left(\mathbb{F}_{v}\right),
$$

where $\mathbb{F}_{v}$ is the residue field of $K_{v}$ and $\overline{\mathcal{A}}_{v}$ is $\mathcal{A}_{v} \times \operatorname{Spec}\left(\mathcal{O}_{v}\right) \operatorname{Spec}\left(\mathbb{F}_{v}\right)$. Since $\overline{\mathcal{A}}_{v}\left(\mathbb{F}_{v}\right)$ is a direct limit of finite groups, we have $\overline{\mathcal{A}}_{v}\left(\mathbb{F}_{v}\right) \otimes \mathbb{Q}_{p} / \mathbb{Z}_{p}=0$. This implies the following assertion:

The map $\alpha$ in the diagram (18) must be surjective.

Consequently, $A\left(K_{v}\right) \otimes \mathbb{Q}_{p} / \mathbb{Z}_{p}$ is a subgroup of $B_{K_{v}}$ for every $v \in S$. This completes the proof of the first assertion of the lemma.

Next, we prove the second assertion. Suppose that $K$ is contained in $F_{\infty}$. Consider the following diagram:

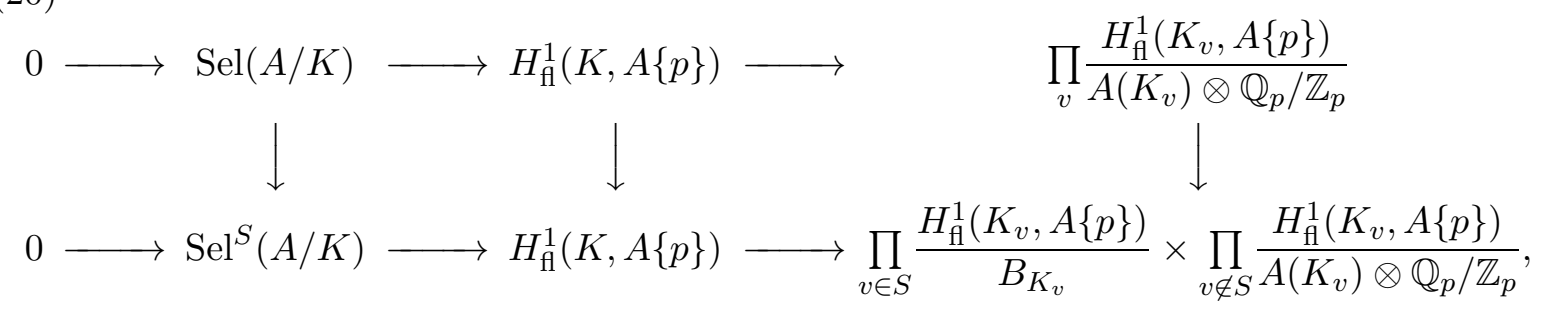

Thus, $\operatorname{Sel}^{S}(A / K) / \operatorname{Sel}(A / K)$ is a subquotient of $\prod_{v \in S} \frac{B_{K_{v}}}{A\left(K_{v}\right) \otimes \mathbb{Q}_{p} / \mathbb{Z}_{p}}$ by the snake lemma. Since $\frac{B_{K_{v}}}{A\left(K_{v}\right) \otimes \mathbb{Q}_{p} / \mathbb{Z}_{p}}$ is a quotient of

$$
C_{K_{v}}:=\operatorname{Coker}\left[\widehat{\mathcal{A}}_{v}\left(\mathcal{O}_{v}\right) \otimes_{\mathbb{Z}} \mathbb{Q}_{p} / \mathbb{Z}_{p} \stackrel{\delta}{\longrightarrow} \underset{n}{\lim } H_{\mathrm{f}}^{1}\left(K_{v}, \widehat{\mathcal{A}}_{v}\left[p^{n}\right]\right)\right],
$$

it suffices to show that $\prod_{v \in S} C_{K_{v}}$ is cofinitely generated of corank less than or equal to $g \sharp S$ over $\mathbb{Z}_{p}$. Let $K_{v}^{\text {ur }}$ be the maximal unramified extension of $K_{v}$. By the Hochshild-Serre spectral sequence, we have the following exact sequence.

$$
\begin{aligned}
0 \longrightarrow \underset{n}{\lim _{n}} H^{1}\left(\Gamma, \widehat{\mathcal{A}}_{v}\left[p^{n}\right]\left(\mathcal{O}_{v}^{\text {ur }}\right)\right) \longrightarrow & \underset{n}{\lim _{\mathrm{fl}}} H_{1}^{1}\left(K_{v}, \widehat{\mathcal{A}}_{v}\left[p^{n}\right]\right) \longrightarrow H_{\mathrm{ff}}^{1}\left(K_{v}^{\mathrm{ur}}, \widehat{\mathcal{A}}_{v}\left[p^{n}\right]\right)^{\Gamma} \\
& \longrightarrow \underset{n}{\lim } H^{2}\left(\Gamma, \widehat{\mathcal{A}}_{v}\left[p^{n}\right]\left(\mathcal{O}_{v}^{\text {ur }}\right)\right) \longrightarrow 0
\end{aligned}
$$


where $\Gamma=\operatorname{Gal}\left(K_{v}^{\mathrm{ur}} / K_{v}\right)$. By the assumption that $A$ is ordinary at $v \in S, \widehat{\mathcal{A}}_{v}\left(\mathcal{O}_{v}^{\text {ur }}\right)$ is isomorphic to $\left(U_{K_{v}^{\text {ur }}}^{1}\right)^{g}$, where $U_{K_{v}^{\text {ur }}}^{1} \subset \mathcal{O}_{K_{v}^{\text {ur }}}^{\times}$is the group of principal units. Since $\widehat{\mathcal{A}}_{v}\left[p^{n}\right]\left(\mathcal{O}_{v}^{\text {ur }}\right)=0$, (22) implies the following isomorphism:

$$
\underset{n}{\lim _{\longrightarrow}} H_{\mathrm{fl}}^{1}\left(K_{v}, \widehat{\mathcal{A}}_{v}\left[p^{n}\right]\right) \cong \underset{n}{\lim _{n}} H_{\mathrm{fl}}^{1}\left(K_{v}^{\mathrm{ur}}, \widehat{\mathcal{A}}_{v}\left[p^{n}\right]\right)^{\Gamma} .
$$

Since $\left(\widehat{\mathcal{A}}_{v}\left(\mathcal{O}_{v}^{\text {ur }}\right)\right)^{\Gamma}=\widehat{\mathcal{A}}_{v}\left(\mathcal{O}_{v}\right)$, we have the following commutative diagram:

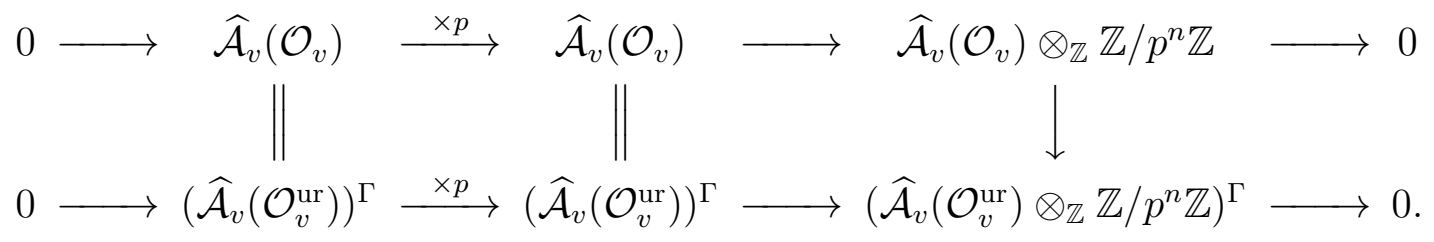

The surjectivity of the last map in the lower line of the diagram follows from the fact that $H^{1}\left(\Gamma, \widehat{\mathcal{A}}_{v}\left(\mathcal{O}_{v}^{\text {ur }}\right)\right)$ is trivial. Hence, we have $\widehat{\mathcal{A}}_{v}\left(\mathcal{O}_{v}\right) \otimes_{\mathbb{Z}} \mathbb{Z} / p^{n} \mathbb{Z} \cong\left(\widehat{\mathcal{A}}_{v}\left(\mathcal{O}_{v}^{\text {ur }}\right) \otimes_{\mathbb{Z}} \mathbb{Z} / p^{n} \mathbb{Z}\right)^{\Gamma}$. Thus, by taking the inductive limit with respect to $n$, we have:

$$
\widehat{\mathcal{A}}_{v}\left(\mathcal{O}_{v}\right) \otimes_{\mathbb{Z}} \mathbb{Q}_{p} / \mathbb{Z}_{p} \cong\left(\widehat{\mathcal{A}}_{v}\left(\mathcal{O}_{v}^{\text {ur }}\right) \otimes_{\mathbb{Z}} \mathbb{Q}_{p} / \mathbb{Z}_{p}\right)^{\Gamma}
$$

We have

$$
\begin{aligned}
& \text { Coker }\left[\widehat{\mathcal{A}}_{v}\left(\mathcal{O}_{v}^{\mathrm{ur}}\right) \otimes_{\mathbb{Z}} \mathbb{Q}_{p} / \mathbb{Z}_{p} \stackrel{\delta}{\longrightarrow} \underset{n}{\lim } H_{\mathrm{f}}^{1}\left(K_{v}^{\mathrm{ur}}, \widehat{\mathcal{A}}_{v}\left[p^{n}\right]\right)\right] \\
& \cong \text { Coker }\left[\left(U_{K_{v}^{\mathrm{ur}}}^{1} \otimes_{\mathbb{Z}} \mathbb{Q}_{p} / \mathbb{Z}_{p}\right)^{g} \longrightarrow H_{\mathrm{fl}}^{1}\left(K_{v}^{\mathrm{ur}},\left(\mu_{p^{n}}\right)^{g}\right)\right] \\
& \cong \text { Coker }\left[\left(U_{K_{v}^{\mathrm{ur}}}^{1 \mathrm{u}} \otimes_{\mathbb{Z}} \mathbb{Q}_{p} / \mathbb{Z}_{p}\right)^{g} \longrightarrow\left(\left(K_{v}^{\mathrm{ur}}\right)^{\times} \otimes_{\mathbb{Z}} \mathbb{Q}_{p} / \mathbb{Z}_{p}\right)^{g}\right] \cong\left(\mathbb{Q}_{p} / \mathbb{Z}_{p}\right)^{g}
\end{aligned}
$$

By (21), (23), (24) and (25),$C_{K_{v}}$ is a subgroup of $\left(\mathbb{Q}_{p} / \mathbb{Z}_{p}\right)^{g}$ for each $v \in S$. This completes the proof of the lemma.

We have the following lemma:

Lemma 4.3. Under the same assumption as that of Theorem 1.9, the group $X^{S}(A / L)_{H} \cong$ $\left(\operatorname{Sel}^{S}(A / L)^{H}\right)^{\vee}$ is a finitely generated $\mathbb{Z}_{p}$-module.

Proof. Let $S$ be a finite set of primes in $F_{\infty}^{(p)}$ where the extension $L / F_{\infty}^{(p)}$ is ramified. Since $A$ has good reduction outside $S$ and since $L / F_{\infty}^{(p)}$ is unramified outside $S$, we have the following commutative diagram:

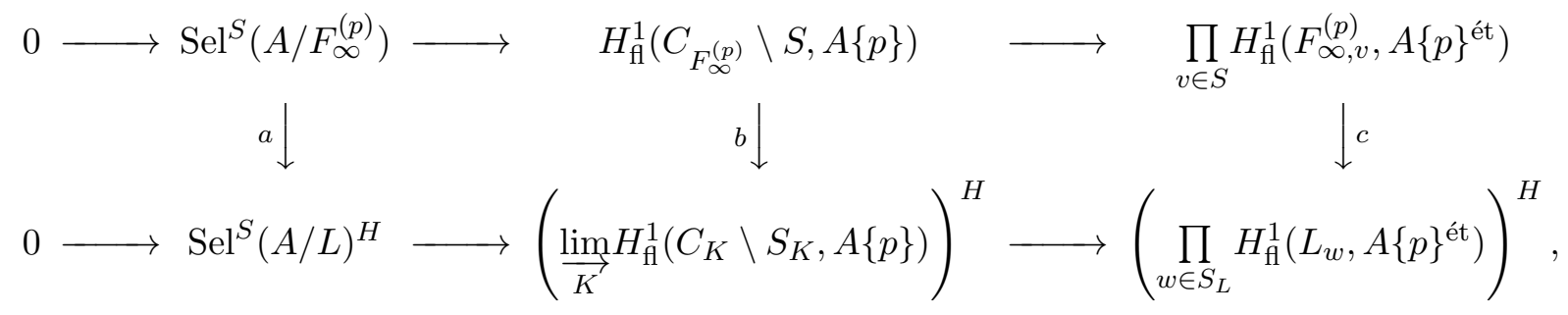

where $K$ runs a finite extension of $F_{\infty}^{(p)}$ contained in $L$ and $S_{K}$ is the set of primes of $K$ lying over $S$. By the Hochshild-Serre spectral sequence, we have the following exact exact 
sequence:

$$
\begin{aligned}
H^{1}(H, A\{p\}(L)) \longrightarrow H_{\mathrm{fl}}^{1}\left(C_{\left.F_{\infty}^{(p)} \backslash S, A\{p\}\right)} \backslash\right. & \\
& \stackrel{b}{\longrightarrow}\left(\underset{F_{\infty}^{(p)} \subset K \subset L}{\lim _{\longrightarrow}} H_{\mathrm{fl}}^{1}\left(C_{K} \backslash S_{K}, A\{p\}\right)\right)^{H} \longrightarrow H^{2}(H, A\{p\}(L)) .
\end{aligned}
$$

Since $H$ is a $p$-adic Lie group and $A\{p\}(L)$ is an abelian group which is cofinite type over $\mathbb{Z}_{p}, H^{i}(H, A\{p\}(L))^{\vee}$ is finitely generated over $\mathbb{Z}_{p}$ for every $i$. In the same way, the Pontrjagin dual of $\operatorname{Ker}(c) \cong \prod_{v \in S_{F_{\infty}^{(p)}}} \prod_{w \mid v} H^{1}\left(H_{w}, A\{p\}^{\text {ét }}\left(L_{w}\right)\right)$ is a finitely generated $\mathbb{Z}_{p}$-module, where $H_{w} \subset H$ is the decomposition subgroup at $w$ of $L$. The module $X\left(A / F_{\infty}^{(p)}\right)=\operatorname{Sel}\left(A / F_{\infty}^{(p)}\right)^{\vee}$ is finitely generated over $\mathbb{Z}_{p}$ by the assumption of Theorem 1.9. By Lemma 4.2, $X^{S}\left(A / F_{\infty}^{(p)}\right)=\operatorname{Sel}^{S}\left(A / F_{\infty}^{(p)}\right)^{\vee}$ is also a finitely generated $\mathbb{Z}_{p}$-module. This completes the proof of Lemma.

Now, we will complete the proof of Theorem 1.9 by using the following result of Balister and Howson:

Lemma 4.4. [B-H, Corollary in $\S 3]$ Let $H$ be a $p$-adic Lie group and $X$ a compact $\Lambda(H)$ module. Let $X_{H}$ denote the $H$-coinvariant quotient of $X$. If $X_{H}$ is a $\mathbb{Z}_{p}$-module of finite type, then $X$ is a $\Lambda(H)$-module of finite type.

In fact, since $X(A / L)_{H}$ is a quotient of $X^{S}(A / L)_{H}, X(A / L)_{H}$ is a finitely generated $\mathbb{Z}_{p}$-module. Hence $X(A / L)$ is a $\Lambda(H)$-module of finite type by using the above lemma.

\section{REFERENCES}

[BBM] P. Berthelot, Pierre; Breen, Lawrence; Messing, William Théorie de Dieudonné cristalline. II, Lecture Notes in Mathematics, 930. Springer-Verlag, 1982.

[B-H] P. Balister, S. Howson, Note on Nakayama's Lemma for Compact-modules, Asian J. Math., Vol. 1, no. 2, 1997.

[CFKSV] J. Coates, T. Fukaya, K. Kato, R. Sujatha, O. Venjakob, The GL $L_{2}$ main conjecture for elliptic curves without complex multiplication, Publ. Math., Inst. Hautes Etud. Sci. 101, 163-208, 2005.

[EL] J. Etesse, B. Le Stum, Fonctions L associées aux F-isocristaux surconvergents II: Zéros et pôles unités, Invent. Math. 127, No.1, 1-31, 1997.

[K] K. Kato, p-adic Hodge theory and values of zeta functions of modular forms, Astérisque 295, 117-290, 2004.

[K-T] K. Kato, F. Trihan, On the conjecture of Birch and Swinnerton-Dyer in characteristic $p>0$, Invent. Math. 153 537-592, 2003.

[L] S. Lang, Algebraic groups over finite fields, American Jour. of Math. 78, 555-563, 1956.

[Mn] Y. Manin, Cyclotomic fields and modular curves (Russian), Uspehi Mat. Nauk 26, no. 6(162), 7-71, 1971.

$[\mathrm{Mz}] \quad$ B. Mazur, Rational points of abelian varieties with values in towers of number fields, Invent. Math. 18, 183-266, 1972.

$[\mathrm{MW}]$ B. Mazur, A. Wiles, Class fields of abelian extensions of $\mathbb{Q}$, Invent. Math. 76, no. 2, 179-330, 1984.

[Mi1] J.S. Milne, Etale cohomology. Princeton Mathematical Series, 33, Princeton University Press, 1980 .

[Mi2] J.S. Milne, Arithmetic duality theorems, Perspectives in Mathematics, 1, Academic Press, 1986.

$[\mathrm{Mu}]$ D. Mumford, Abelian varieties, Tata Institute of Fundamental Research Studies in Mathematics, No. 5, 1970. 
[R] K. Rubin, The "main conjectures" of Iwasawa theory for imaginary quadratic fields, Invent. Math. 103, 25-68, 1991.

[S] P. Schneider, p-adic height pairing. II, Invent. Math. 79, 329-374, 1985.

[T] T. Tsuji, Poincaré duality for logarithmic crystalline cohomology Compositio Math. 118, no. 1, 11-41. 1999

[We] A. Weil, Basic number theory, Springer-Verlag, 1973.

[W] A. Wiles, The Iwasawa conjecture for totally real fields, Ann. of Math. (2) 131, no. 3, 493-540, 1990. 